\title{
Pyruvate-Dependent Changes in Neutrophil Amino- and Alpha-Keto Acid Profiles or Immunity: Which Mechanisms Are Involved?
}

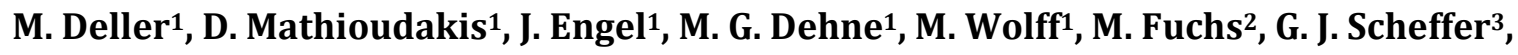 \\ J. Mühling ${ }^{*}$ \\ ${ }^{1}$ Clinics of Anaesthesiology, Intensive Care Medicine and Pain Therapy, University Hospital Giessen and \\ Marburg, Justus-Liebig-University, Giessen, Germany \\ ${ }^{2}$ Dr. Ing. Herbert Knauer GmbH, Berlin, Germany \\ ${ }^{3}$ Department of Anesthesiology, Pain, and Palliative Medicine, Radboud University Medical Center, Nijmegen, \\ The Netherlands \\ Email: ${ }^{\text {j.muhling@anes.umcn.nl, jorg.muhling@radboudumc.nl }}$
}

Received 9 September 2014; revised 9 October 2014; accepted 9 November 2014

Copyright (C) 2014 by authors and Scientific Research Publishing Inc.

This work is licensed under the Creative Commons Attribution International License (CC BY).

http://creativecommons.org/licenses/by/4.0/

c) (i) Open Access

\begin{abstract}
High current findings indicate that a substitution with pyruvate can lead to significant alterations or even improvement in neutrophil immunonutrition. However, it is still unknown which intracellular pathways might be involved here. Hence, in this study, we investigated whether preincubation with an inhibitor of •NO-synthase (L-NAME), an •NO donor (SNAP), an analogue of taurine (beta-alanine), an inhibitor of ornithine-decarboxylase (DFMO) as well as a glutamine-analogue (DON), is able to alter the intragranulocytic metabolic response to pyruvate, here for example studied for neutrophil intracellular amino- and $\alpha$-keto acid concentrations or important neutrophil immune functions [released myeloperoxidase (MPO), the formation of superoxide anions $\left(\mathrm{O}_{2}^{-}\right)$ and hydrogen peroxide $\left(\mathrm{H}_{2} \mathrm{O}_{2}\right)$ ]. In summary, the interesting first results presented here showed, that any damage of specific metabolic pathways or mechanisms, which seem directly or indirectly to be involved in relevant pyruvate dependent granulocytic nutrient content or specific cellular tasks, could lead to therapeutically desired, but also to unexpected or even fatal consequences for the affected cells. We therefore continue to believe that pyruvate, irrespective of which exact biochemical mechanisms were involved, in neutrophils may satisfy the substantial metabolic demands for a potent intracellular nutrient.
\end{abstract}

\section{Keywords}

Pyruvate, DON, L-NAME, SNAP, $\beta$-Alanine, DFMO, Neutrophil, Amino Acids, $\alpha$-Keto Acids, Immune

\footnotetext{
*Corresponding author.
}

How to cite this paper: Deller, M., Mathioudakis, D., Engel, J., Dehne, M.G., Wolff, M., Fuchs, M., Scheffer, G.J. and Mühling, J. (2014) Pyruvate-Dependent Changes in Neutrophil Amino- and Alpha-Keto Acid Profiles or Immunity: Which Mechanisms Are Involved? Open Journal of Immunology, 4, 157-174. http://dx.doi.org/10.4236/oji.2014.44018 


\section{Function, Immunonutrition}

\section{Introduction}

Neutrophil granulocytes form an indispensable component of the innate immune system and are the most abundant type of white blood cells in mammals, accounting for close to $60 \%-70 \%$. Being highly motile, they quickly congregate at a focal point of infection, attracted by cytokines expressed by activated endothelial and various other cells. After activation, neutrophils express and release cytokines, which in turn amplify inflammatory reactions, and are capable of attacking microorganisms directly by use of three impressive weapons: phagocytosis, release of soluble anti-microbials and generation of neutrophil extracellular fibre traps.

However, the successful achievement of these tasks depends on one key requirement: the subtle and the especially undisturbed interplay of all relevant intracellular biochemical pathways. In fact, the cell is obviously equipped with an impressive arsenal of metabolic pathways capable of taking on the role of a highly effective immunological weapon. Examples here include the reversible transamination of pyruvate by the alanine aminotransferase (producing alanine and $\alpha$-ketoglutarate), the anaplerotic carboxylation of pyruvate metabolized by pyruvate carboxylase (which provides oxalacetate precursors for the citric acid cycle and gluconeogenesis), the "de novo synthesis" of important sugars, amino and $\alpha$-keto acids (i.e. glucose, alanine, $\alpha$-ketoglutarate, arginine, ornithine, etc.) or the ability to form energy-rich molecules such as nicotinamide adenine dinucleotide phosphate (NADPH) or guanosine-5'-triphosphate (GTP) from pyruvate-depent pathways, and so on [1]-[6]. Simply one of the most important biochemical processes in which pyruvate is involved, the conversion of pyruvate by the pyruvate dehydrogenase complex (PDH) to form acetyl-CoA, an important link substrate between the metabolic pathways of glycolysis and the citric acid cycle, was, actually and not really surprising to the concerned viewer, also found in neutrophils [7]-[10].

So it is not surprising that pyruvate, or simple pyruvate-dependent derivatives (i.e. ethyl-pyruvate) which are preferred due to known galenic problems in vivo, is moved as a potential metabolic substrate into the focus of scientific immunonutrition research. And observing the first interesting results, there seems to be little doubt: pyruvate, whose name is derived from the greek "pyr" (fire, heat) and the latin "uva” (grape), indeed, impressively fulfills the criteria for a potent molecule in modulation of endogenous immunoregulation and also seems to play a relevant role in granulocytic host defence mechanisms, of course taking advantage of existing extraand intracellular $\alpha$-keto and amino acid pathways [11]-[13].

But if one looks, however, at the current results available so far, another fact is unfortunately recognizable: detailed studies about possible immunonutritional effects of pyruvate or pyruvate-dependent derivatives of the amino or $\alpha$-keto acid content or metabolism in neutrophils or are not yet available [5] [14]. An example is glutamine metabolism in neutrophils. Here it remains entirely unclear whether an inhibitor of glutamine-requiring enzymes may especially influence pyruvate-induced metabolic and immunological effects [15]. The same applies to other metabolic pathways which are particularly significant for neutrophils: for example the question whether the effects of pyruvate on neutrophil metabolism and immune functionality are modulated by alterations in intracellular amino or $\alpha$-keto acid pathways, which are related with nitric oxide $(\cdot \mathrm{NO})$, ornithine, arginine or taurine content, metabolism or transport [11] [16]-[21].

The design of our new study was therefore to document and compare the effects of various metabolic modulators on neutrophil amino acid and $\alpha$-ketoacid concentrations or important neutrophil immune functions [released myeloperoxidase (MPO), the formation of superoxide anions $\left(\mathrm{O}_{2}^{-}\right)$and hydrogen peroxide $\left.\left(\mathrm{H}_{2} \mathrm{O}_{2}\right)\right]$, in order to investigate which pathways may be affected in a pyruvate-induced immunonutrition of neutrophils. In our study, it was of particular importance, that the metabolic modulators were preinvestigated adequately. For this reason we selected the following: $\mathrm{N}_{\omega}$-nitro-L-arginine-methylester-hydrochloride [L-NAME, inhibitor of •NO-synthase], S-nitroso-N-acetyl-penicillamine [SNAP, •NO donor], 6-diazo-5-oxo-L-norleucine [DON, glutamine-analogue], $\beta$-alanine [ $\beta$-Ala, taurine-analogue] and $\alpha$-difluoro-methyl-ornithine [DFMO, inhibitor of ornithine-decarboxylase].

\section{Materials and Methods}

The study and the consent procedure was approved by the local ethics committee of the Justus Liebig University 
Giessen, Germany (No. 69/99). The blood samples were taken in healthy volunteers, which appeared only for this cause in our research laboratory, and both the verbal consent as well as the personal and biometric data were accurately documented. After completion of the studies the volunteers received their test results with the screening of intra- and extracellular amino- and $\alpha$-keto acids for their own medical documentation. Ten adult males between 25 and 46 years (34 \pm 9.1 ) with an average height of $177.8 \mathrm{~cm}$ (range $171-188$ ) and weight of 81.7 kg (range 72 - 96) were selected. Those men with metabolic (e.g. diabetes, etc.), cardiopulmonary, neurological or allergic diseases or men taking drugs were excluded. Whole blood samples (lithium-heparinate plastic tubes) were withdrawn between 08:00 and 09:00 (after 10 hours of fasting) with consideration of circadian variations. $\mathrm{P} \leq 0.05$ was considered statistically significant.

\subsection{Pyruvate (PYR) Combined with L-NAME, SNAP, DON, $\beta$-Ala or DFMO}

Whole blood samples were incubated with aqueous solutions of pyruvate (PYR, $1 \mathrm{mM}$, Fresenius, Bad Homburg, Germany) stabilized conscientiously for medical use due to known galenic problems (pH 4.5, $\mathrm{Na}^{+}$: $20 \mathrm{mM}$ [22]) as well as with PYR $(1 \mathrm{mM})+$ L-NAME ( $1 \mathrm{mM}, \mathbf{N}_{\omega}$-nitro-L-arginine-methylester-hydrochloride; inhibitor of nitric oxide $(\cdot N O)$ synthase; Calbiochem, Bad Schwalbach, Germany), PYR $(1 \mathrm{mM})+$ SNAP $(100 \mu M$, S-nitroso-N-acetyl-penicillamine; exogenous nitric oxide donor ( $\bullet$ NO-release: $5.6 \mu \mathrm{M} / \mathrm{min})$; Sigma, Deisenhofen, Germany), PYR (1 mM) + DON (100 $\mu$ M, 6-diazo-5-oxo-L-norleucine; analogue of glutamine and inhibitor of glutamine-requiring enzymes; Sigma, Deisenhofen, Germany), PYR (1 mM) + $\boldsymbol{\beta}$-Ala (10 mM, $\beta$-alanine; analogue of taurine and taurine transport antagonist; Sigma, Deisenhofen, Germany) or PYR (1mM) + DFMO (1 mM, $\alpha$-difluoro-methyl-ornithine; irreversible inhibitor of ornithine decarboxylase; Sigma, Deisenhofen, Germany). For the latter PYR was added after a 15-minute pre-incubation of L-NAME, SNAP, DON, $\beta$-Ala or DFMO, this mixture was incubated again for another 120 minutes. The selected PYR concentration corresponded to former pyruvate incubation in vitro results [12] [23].

All solutions were prepared and diluted in Hank's balanced salt solution (HBSS; Sigma, Deisenhofen, Germany) and the $\mathrm{pH}$ in the test solution was confirmed to be 7.4. One milliliter of whole blood was incubated with $25 \mu \mathrm{l}$ of test solution (final pyruvate concentrations were as described above) at $37^{\circ} \mathrm{C}$ using a vibrating water bath. Corresponding volumes of HBSS were added to the control tubes. Before further processing all fractions were immediately cooled in an ice water bath at $4^{\circ} \mathrm{C}$ and $100 \mu \mathrm{g} / \mathrm{ml}$ phenyl methyl sulfonyl fluoride (PMSF), 10 $\mu \mathrm{g} / \mathrm{ml}$ leupeptin, $10 \mu \mathrm{g} / \mathrm{ml}$ pepstatin, as well as $10 \mu \mathrm{g} / \mathrm{ml}$ antipain (all acquired from Sigma, USA) were added to each plastic heparin tube before the blood samples; these additions served to inhibit proteases.

\subsection{Highly Selective Separation of PMN from Whole Blood}

Precise details of our PMN-separation technique have been described previously. This method allows a very rapid and selective enrichment of neutrophils while preserving high cellular viability and integrity from very small quantities of whole blood [24]. Separation of PMN was accomplished using a cooled $\left(4^{\circ} \mathrm{C}\right)$ Percoll ${ }^{\circledR}$ gradient (Pharmacia, Uppsala, Sweden). $4 \mathrm{ml}$ portions $(\Sigma=12 \mathrm{ml}$ ) of cooled whole blood from each volunteer were overlaid onto previously prepared and precooled $\left(4^{\circ} \mathrm{C}\right.$ ) $70 \% / 55 \%$ (in $0.9 \% \mathrm{NaCl}$ ) Percoll ${ }^{\circledR}$-gradients before centrifugation at $350 \times \mathrm{g}$ for 15 minutes at $4^{\circ} \mathrm{C}$ (Biofuge ${ }^{\circledR}$, Heraeus, Hanau, Germany). This separates the PMN as a small layer between the erythrocyte and monocyte layers. The PMN were carefully removed from the sample and suspended in $10 \mathrm{ml}$ cooled $\left(4^{\circ} \mathrm{C}\right)$ phosphate buffered saline (PBS) stock buffer (diluted 1:10, v/v; $10 \times$ PBS stock buffer, without $\mathrm{Ca}^{2+} / \mathrm{Mg}^{2+}$, Gibco, Karlsruhe, Germany). After a second centrifugation step (350 $\times \mathrm{g}$ for $5 \mathrm{~min}$ at $4^{\circ} \mathrm{C}$ ), the PBS buffer was discarded and the erythrocytes remaining in the sample were hypotonically lysed using $2 \mathrm{ml}$ of cooled $\left(4^{\circ} \mathrm{C}\right)$ distilled water (Pharmacia, Uppsala, Sweden). After 20 seconds the PMN fraction was immediately brought back to isotonicity by the addition of $1 \mathrm{ml}$ of $2.7 \% \mathrm{NaCl}$ (Merck, Darmstadt, Germany) at $4^{\circ} \mathrm{C}$ and resuspended by adding $10 \mathrm{ml}$ of diluted stock PBS buffer. After a third centrifugation step (350 $\times \mathrm{g}$ for $5 \mathrm{~min}$ at $4^{\circ} \mathrm{C}$ ) the PBS buffer was discarded and the PMN fraction again resuspended (200 $\mu \mathrm{l} \mathrm{PBS}$ buffer). Subsequently, all PMN fractions were combined and two aliquots of resuspended sample were removed for microscopy. On average, the cell fractionation procedure lasted $34 \pm 4$ min. Immediately after preparation, the extracted PMN samples were frozen at $-80^{\circ} \mathrm{C}$ before lyophilization (freeze dryer CIT- ${ }^{\circledR}$, Heraeus, Hanau, Germany). These conditions allowed for a PMN lysis which was not chemically mediated and guaranteed longer analyte stability during extended storage of the sample. Samples prepared in this manner were stored at $-80^{\circ} \mathrm{C}$ until analyzed within a period not exceeding four weeks. The purity, determined in duplicate in the first aliquot 
by dyeing with “Türk's Solution ${ }^{\circledR}$ " (Merck) and viability, determined in the second aliquot by exclusion of "Trypan Blue ${ }^{\circledR}$ " (Merck) were examined and verified by light microscopy (Zeiss, Oberkochen, Germany). Cell yields were determined at the same time that viability was measured, samples with a PMN purity and viability < 96\% were discarded. In parallel, plasma samples (100 $\mu \mathrm{l})$ were separated, lyophilized and stored using known techniques.

\subsection{Chromatographic Amino and $\alpha$-Keto Acid Analysis}

Amino and $\alpha$-keto acids in PMN were quantified using previously described methods which fulfilled the strict criteria required for ultrasensitive, comprehensive amino acid and $\alpha$-keto acid analysis, specially developed and precisely validated in our institute for this purpose [14] [24] [25]. Moreover, the coefficients of variations for both method reproducibility and reproducibilities of retention times were also within normal ranges. PMN amino acid concentrations are given in $10^{-16}$ moles per neutrophil-cell, while PMN $\alpha$-keto acid concentrations are given in $10^{-17}$ moles per neutrophil-cell.

\subsection{Preparation of Derivatization Reagent}

For the fluorescence labeling of the $\alpha$-keto acids, we used $o$-phenylenediamine (OPD, Sigma, Deisenhofen, Germany). Since oxidation of OPD influences the results in a negative way (the oxidized reagent causes variation in the fluorescence intensity) the brown powder must be re-crystallized prior to use. Although the amount of reactive OPD is less when using the oxidized form of the reagent, this re-crystallization procedure is necessary even when starting with the originally supplied substance. The $o$-phenylenediamine was dissolved in heptane at a temperature of $100^{\circ} \mathrm{C}-120^{\circ} \mathrm{C}$ (oil bath, Merck) and the heptane subsequently evaporated in a rotary evaporator (Merck). This procedure yielded a white powder after drying. With storage under $\mathrm{N}_{2}$ (Sigma, Deisenhofen, Germany) and at $4^{\circ} \mathrm{C}$ in a dark bottle, the dry substance is useable for several months. For each batch of analyses, the OPD reagent must be freshly prepared. For each sample, $5 \mathrm{mg}$ of OPD was dissolved in $5 \mathrm{ml}$ of $3 \mathrm{M} \mathrm{HCl}$ (Sigma) and $10 \mu \mathrm{l}$ of 2-mercaptoethanol (Sigma) was added to yield OPD-HCl-ME. This reagent solution was stable for several hours without loss of sensitivity.

\subsection{Standard Samples \& Precolumn Derivatization Procedure}

Analytically pure $\alpha$-keto acids (Sigma) were dissolved in distilled $\mathrm{H}_{2} \mathrm{O}$ (Merck) containing 4\% human serum albumin (Merck), immediately lyophilized and stored at $-80^{\circ} \mathrm{C}$. The lyophilizates (PMN, plasma and standard samples) were solubilized in $250 \mu \mathrm{l}$ of pure methanol (Mallinckrodt Baker B.V., Deventer, Holland). The methanol also contained the $\alpha$-keto acid, $\alpha$-ketovalerate (KV; Sigma) as an HPLC internal standard. KV is a non-physiological $\alpha$-keto acid. After a 3-minute incubation and a 3-minute centrifugation step (3000 ×g, Rotix$\mathrm{a} / \mathrm{KS}^{\circledR}$, Tuttlingen, Germany), $200 \mu \mathrm{l}$ of the extracts were dried under $\mathrm{N}_{2}\left(10 \mathrm{~min}, 20^{\circ} \mathrm{C}\right.$, Messer, Griesheim, Germany). The OPD-HCl-ME reagent (5 ml) was then added, and the samples were incubated for 60 minutes at $80^{\circ} \mathrm{C}$. The derivatization was stopped after exactly 60 minutes by cooling for 15 minutes in ice water. Ethyl acetate ( $2 \mathrm{ml}$, Sigma) was added to the samples and mixed for 7 minutes in a rotary mixer (Merck) to extract the $\alpha$-keto acids. After extraction, the top ethyl acetate layer was then transferred to a glass vial (2-CRV ${ }^{\circledR}$, Chromacoll, Trumbull, USA). This procedure was repeated twice for each sample. The combined etylaceate portions were dried under $\mathrm{N}_{2}$ (30 min), re-solubilized in $120 \mu \mathrm{l}$ of methanol and $50 \mu \mathrm{l}$ of this mixture was injected onto the HPLC column.

\subsection{Fluorescence High-Performance Liquid Chromatography}

The fluorescence high-performance liquid chromatography system (F-HPLC) consisted of a pump with a controller for gradient programming (600 $\mathrm{E}^{\circledR}$, Waters, Milford, MA, USA) and a programmable autosampler (Triathlon $^{\circledR}$, Spark, Netherlands) with a Rheodyne injection valve and a $100 \mu \mathrm{l}$ sample loop (AS $300^{\circledR}$, Sunchrom, Friedrichsdorf, Germany). A Nova-Pak ${ }^{\circledR}, 300 \times 3.9$ mm i.d., RP-C-18, 60A, $4 \mu \mathrm{m}$ (Waters) analytical column was used for separation. Column temperatures were maintained at $35^{\circ} \mathrm{C}$ using a column oven (Knauer, Berlin, Germany). The column eluent was monitored using a fluorescence spectrophotometer (RF-530 ${ }^{\circledR}$, Shimadzu, Kyoto, Japan) at an excitation wavelength of $360 \mathrm{~nm}$ and an emission wavelength of $415 \mathrm{~nm}$. Data recording and evaluation was performed using computer integration software (EuroChrom $2000^{\circledR}$, Knauer, Berlin, Ger- 
many). The linear calibration curves were constructed based on area ratios of the standard (St) to the sample (S) chromatogramms ([area $a_{\text {keto acid-st }} /$ area $\left._{\text {internal standard-st }}\right] \times$ amount or concentration of keto acids injected $=$ calculation factor (CF); [area keto acid-s $/$ area $\left._{\text {internal standard-s }}\right] \times \mathrm{CF}=$ final result). The flow rate was maintained at $1.0 \mathrm{ml} / \mathrm{min}$ throughout. For the gradient program and solvents, these were automatically degassed using a 3-channel degasser (Knauer, Berlin, Germany).

\subsection{Superoxide Anion Production}

Superoxide anion and hydrogen peroxide production as well as activity of released myeloperoxidase were determined photometrically using modifications of known methods validated in our institute for this purpose [14] [24] [25]. Superoxide anion production was measured by reduction of cytochrome C, $100 \mathrm{mg}$ of cytochrome C (type IV, Sigma, Deisenhofen, Germany) which was dissolved in $30 \mathrm{~mL} \mathrm{PBS}{ }^{\circledR}$-glucose buffer. The solution was aliquoted and frozen at $-20^{\circ} \mathrm{C}$. Opsonized zymosan (Sigma, Deisenhofen, Germany) was used to stimulate PMN. It was produced by incubating $100 \mathrm{mg}$ zymosan with $6 \mathrm{~mL}$ pool serum for $30 \mathrm{~min}$ at $37^{\circ} \mathrm{C}$. After washing with saline and centrifuging centrifugation at $350 \times \mathrm{g}(10 \mathrm{~min})$ opsonized zymosan was re-suspended in $10 \mathrm{~mL} \mathrm{PBS}^{\circledR}$ glucose buffer, aliquoted and frozen at $-20^{\circ} \mathrm{C}$. The PMN were then isolated using a modification of our PMNseparation technique (as mentioned above). After stepwise (15 minutes and 5 minutes) centrifugation procedures $\left(350 \times \mathrm{g}, 20^{\circ} \mathrm{C}\right)$ as well as careful lysis of a few erythrocytes contaminating the pellet, the pelleted PMN- cells were re-suspended by adding diluted PBS ${ }^{\circledR}$ (Gibco, Karlsruhe, Germany) stock buffer. After 7 mL PBS ${ }^{\circledR}$ stock buffer had been administered, the tube was centrifuged at $350 \times \mathrm{g}$ for 5 minutes $\left(20^{\circ} \mathrm{C}\right)$. The supernatant was decanted. Samples with a PMN purity $<96 \%$ and those with more than $4 \%$ of dead cells were discarded. The PMN concentration required in each case was adjusted by adding PBS containing 9.99 g glucose (Merck, Darmstadt, Germany). After PMN isolation, $500 \mu \mathrm{L}$ zymosan, $150 \mu \mathrm{L}$ pool serum, $250 \mu \mathrm{L}$ cytochrom C and $500 \mu \mathrm{L}$ isolated PMN suspension $\left(0.8 \times 10^{6} / \mathrm{mL}\right)$ and again the pyruvate to be tested, were poured into a test tube. A preparation containing $500 \mu \mathrm{L}$ buffer instead of zymosan was used as a zero adjustment. After further incubation for 15 minutes at $37^{\circ} \mathrm{C}$ the reaction was stopped by putting the test tube into ice water. After centrifugation $(350 \times \mathrm{g}$; $5 \mathrm{~min}, 4^{\circ} \mathrm{C}$ ) extinction of the supernatant was measured photometrically $\left(546 \mathrm{~nm}\right.$; Digitalphotometer $6114 \mathrm{~S}^{\circledR}$; Eppendorf, Germany). The amount of superoxide anion produced was calculated and resulted from the extinction coefficient of cytochrome $\mathrm{C}$ according to the Lambert-Beer law. All control probes obtained for standard curves have been prepared, incubated and measured identically.

\subsection{Hydrogen Peroxide Production}

Hydrogen peroxide production was also determined photometrically. The method was based on horseradish peroxidase catalysed by oxidation of phenol red by hydrogen peroxide [14] [24] [25]. Phenol red (Sigma, Deisenhofen, Germany) and horseradish peroxidase (type II, Sigma, Deisenhofen, Germany) were added to PMN which had been stimulated by opsonized zymosan. Phenol red was dissolved in double-distilled water (10 g/L). Horseradish peroxidase was dissolved in $\mathrm{PBS}^{\circledR}$-glucose buffer $(5 \mathrm{~g} / \mathrm{L})$. After incubation of whole blood with pyruvate at $37^{\circ} \mathrm{C}$, PMN were isolated as described above. Isolated PMN were stimulated by opsonized zymosan (Sigma, Deisenhofen, Germany). The final test preparation consisted of $500 \mu \mathrm{L}$ zymosan, $125 \mu \mathrm{L}$ pool serum, $12.5 \mu \mathrm{L}$ horseradish peroxidase, $12.5 \mu \mathrm{L}$ phenol red, $12.5 \mu \mathrm{L}$ sodium azide $(200 \mathrm{mmol} / \mathrm{L} / \mathrm{l}$; Merck, Darmstadt, Germany), $500 \mu \mathrm{L}$ PMN suspension $\left(2 \times 10^{6} \mathrm{PMN}-\mathrm{cells} / \mathrm{mL}\right)$ and again pyruvate. After incubation for $15 \mathrm{mi}-$ nutes $\left(37^{\circ} \mathrm{C}\right)$, the test preparation was centrifuged for 5 minutes $\left(350 \times \mathrm{g} ; 4^{\circ} \mathrm{C}\right)$. Subsequent to adding $25 \mu \mathrm{L}$ sodium hydroxide solution (1.0 normal, Merck, Darmstadt, Germany) the extinction was measured photometrically at $623 \mathrm{~nm}$. All control probes obtained for standard curves have been prepared, incubated and measured identically.

\subsection{Activity of Released Myeloperoxidase}

Activity of released myeloperoxidase was also determined photometrically [14] [24] [25]. 1 mmol/L 2.2'-azino-di-(3-ethylbenzthiazoline) sulphonic acid (ABTS, Sigma, Deisen-hofen, Germany) was dissolved in 0.1 $\mathrm{Mol} / \mathrm{L}$ citrate buffer (Behring, Marburg, Germany; $\mathrm{pH}$ 7.4). After incubation of whole blood with pyruvate at $37^{\circ} \mathrm{C}, 100 \mu \mathrm{L}$ isolated PMN suspension $\left(2 \times 10^{6} / \mathrm{mL}\right)$ was incubated with $0.5 \mu$ g cytochalasin B (Sigma, Deisenhofen, Germany) and again with pyruvate $\left(5 \mathrm{~min} ; 37^{\circ} \mathrm{C}\right)$. After adding $100 \mu \mathrm{L}$ opsonized zymosan and sup- 
plementing in order to keep the concentration constant, the preparation was incubated again for $10 \mathrm{~min}\left(37^{\circ} \mathrm{C}\right)$. Then $1 \mathrm{~mL}$ ATBS solution was added. After centrifuging $\left(700 \times \mathrm{g}, 5 \mathrm{~min}, 20^{\circ} \mathrm{C}\right) 1 \mathrm{~mL}$ of supernatant was removed and mixed with $1 \mu \mathrm{L}$ hydroxide peroxide solution (30\%; Merck, Darmstadt, Germany) and extinction was measured photometrically (405 nm).

\subsection{Statistical Analysis}

Statistical analysis and interpretation of the results were performed in close co-operation with colleagues from the Department of Medical Statistics, Justus Liebig University Giessen, Germany. All tests were performed in duplicate. Thus our PMN amino acid results represent the mean of two estimations. After the results were demonstrated to be normally distributed (Pearson-Stephens test), statistical methods were performed including Bartlett's test to check homogeneity of variance $(\mathrm{p} \leq 0.1)$. If the requirements were met, ANOVA analysis was conducted. If the requirements were not fulfilled, the Friedmann test was performed. Probability levels of $\mathrm{p} \leq$ 0.05 versus control were considered as significant. The data are given as arithmetic means \pm standard deviations (mean $\pm \mathrm{SD}$ ).

\section{Results}

The free intracellular amino and $\alpha$-keto acid concentrations, superoxide anion formation, hydrogen peroxide generation as well as the activity of released myeloperoxidase obtained in the control cells were within normal physiological ranges [14] [24] [25].

An overview of the intracellular amino and $\alpha$-keto acid as well as plasma levels, which were found in the present survey are presented in Table 1. For example intracellular pyruvate levels amounted on average to 6.72 $\times 10^{-17}$ moles per neutrophil (PMN) cell (169.1 $\mu \mathrm{mol} / \mathrm{L}$ PMN cell volume [NCV]; plasma: $\left.102.9 \pm 42.7 \mu \mathrm{mol} / \mathrm{L}\right)$. The intracellular $\alpha$-ketoglutarate content at almost $1.21 \times 10^{-17} \mathrm{~mol}$ per PMN cell $(30.5 \mu \mathrm{mol} / \mathrm{L} \mathrm{NCV}$; plasma: $6.8 \pm 2.3 \mu \mathrm{mol} / \mathrm{L}$ ) was approximately 5.6 times lower. However, the composition of these free amino acid and $\alpha$-keto acid pools does not appear to be arbitrary in any way. When comparing the intra versus the extracellular concentration gradient [i:e], the results painted a very different picture: for pyruvate (i:e: 1.6), glutamine (i:e: 1.2), alanine (i:e: 1.3 ), arginine (i:e: 0.9 ), serine (i:e: 4.1 ) and glycine (i:e: 2.7$)$ not only high plasma but also high intracellular concentrations have been found, while $\alpha$-ketoglutarate (i:e: 4.5), glutamate (i:e: 40.6), aspartate (i:e: 56.8), asparagine (i:e: 1.7), ornithine (i:e: 2.3) with low plasma concentrations apparently accumulated within the neutrophils (Table 1).

\subsection{Effects of PYR Combined with L-NAME, SNAP, DON, $\beta$-Ala or DFMO on the Free $\alpha$-Keto Acid Pools in Neutrophils (Figure 1)}

Additional application of SNAP, DON, $\beta$-alanine or DFMO to PYR also induced important PYR-independent effects. While L-NAME induced no effect exceeding that of singular PYR, significant reductions in $\alpha$-ketoglutarate (PYR: $+115 \%$; PYR + SNAP: $+62 \%$ ) and pyruvate (PYR: $+205 \%$; PYR + SNAP: $+93 \%$ ) that were both clearly raised by singular PYR occurred after SNAP application, although these values remained significantly above the respective control values.

Upon DON application, however, there were significant decreases in all measured $\alpha$-ketoacids that were below the control findings ( $\alpha$-ketoglutarate: PYR + DON: $-26 \%$, pyruvate: PYR + DON: $-31 \%$ and $\alpha$-ketobutyrate: PYR + DON: $-36 \%)$.

Only with the cellular $\alpha$-ketobutyrate content did $\beta$-ala induce additive effects that exceeded singular PYR (PYR: $+22 \%$; PYR $+\beta$-ala: $+61 \%$ ).

DFMO added to PYR induced a decrease in intragranulocytic $\alpha$-ketoglutarate (PYR + DFMO versus PYR: $-34 \%$ ) and also pyruvate (PYR + DFMO versus PYR: $-47 \%)$.

\subsection{Effects of PYR Combined with L-NAME, SNAP, DON, $\beta$-Ala or DFMO on the Free Amino Acid Pools in Neutrophils (Figures 2-8)}

The incubation of PYR with the above described "metabolism modulators" L-NAME, SNAP, DON, $\beta$-Ala or DFMO led, unlike singular application, to significant and decisive findings.

Combined administration of L-NAME and PYR induced no effect that exceeded singular application of PYR. 
Table 1. Content of free amino acids $\left[10^{-16} \mathrm{Mol}\right]$ and $\alpha$-keto acids $\left[10^{-17} \mathrm{Mol}\right]$ per neutrophil (PMN, mean \pm standard deviation [SD]; $\mathrm{n}=10$ ) and their intracellular concentrations based on the mean neutrophil cell volume $(\mathrm{NCV})[\mu \mathrm{Mol} / 1$, mean $\pm \mathrm{SD} ; \mathrm{n}=10]$. NCV was calculated as follows: $\mathrm{NCV}=$ leukocrit $_{\text {sample }} \times \mathrm{PMN}$-number $^{-1}$ $\left[\mathrm{NCV}_{\text {amino acids }}: 431.8 \pm 211.2 \times 10^{-15} \mathrm{~L}, \mathrm{NCV}_{\alpha \text {-keto acids }}: 397.3 \pm 234.8 \times 10^{-15} \mathrm{~L}\right]$. Also pictured are the plasma concentrations found in this study $[\mu \mathrm{Mol} / 1$, mean $\pm \mathrm{SD} ; \mathrm{n}=10]$. Additionally, the intra (I) versus extracellular (E) concentration gradients (I:E) have also been computed.

\begin{tabular}{|c|c|c|c|c|c|c|c|c|}
\hline \multirow{2}{*}{ Amino acids } & & \multicolumn{2}{|c|}{ PMN } & \multicolumn{2}{|c|}{ NCV } & \multicolumn{2}{|c|}{ Plasma } & Gradient \\
\hline & & \multicolumn{2}{|c|}{$10^{-16} \mathrm{Mol} /$ cell } & \multicolumn{2}{|c|}{ [ $\mu \mathrm{Mol} / \mathrm{l}]$} & \multicolumn{2}{|c|}{ [pMol/l] } & I:E \\
\hline \multirow[b]{2}{*}{ aspartate } & \multirow[b]{2}{*}{ (asp) } & \multicolumn{2}{|c|}{ mean \pm SD } & \multicolumn{2}{|c|}{ mean $\pm \mathrm{SD}$} & \multicolumn{2}{|c|}{ mean \pm SD } & \multirow[b]{2}{*}{56.8} \\
\hline & & \multirow{2}{*}{$\begin{array}{l}2.97 \\
6.17\end{array}$} & \multirow{2}{*}{$\begin{array}{l}1.14 \\
1.93\end{array}$} & \multirow{2}{*}{$\begin{array}{c}687.8 \\
1428.9\end{array}$} & \multirow{2}{*}{$\begin{array}{l}264.0 \\
447.0\end{array}$} & \multirow{2}{*}{$\begin{array}{l}12.1 \\
35.2\end{array}$} & \multirow{2}{*}{$\begin{array}{c}4.4 \\
14.9\end{array}$} & \\
\hline glutamate & (glu) & & & & & & & 40.6 \\
\hline asparagine & (asn) & 0.41 & 0.15 & 95.7 & 34.7 & 57.8 & 16.6 & 1.7 \\
\hline serine & (ser) & 2.05 & 0.54 & 474.8 & 125.1 & 115.2 & 39.1 & 4.1 \\
\hline glutamine & $(g \ln )$ & 3.27 & 0.98 & 757.3 & 227.0 & 621.6 & 234.1 & 1.2 \\
\hline histidine & (his) & 1.03 & 0.56 & 238.5 & 129.7 & 78.5 & 27.0 & 3.0 \\
\hline glycine & (gly) & 2.59 & 0.75 & 599.8 & 173.7 & 226.3 & 71.8 & 2.7 \\
\hline threonine & (thr) & 0.78 & 0.26 & 180.6 & 60.2 & 101.4 & 68.4 & 1.8 \\
\hline citrulline & (cit) & 0.13 & 0.04 & 30.1 & 9.3 & 29.5 & 11.2 & 1.0 \\
\hline arginine & (arg) & 0.34 & 0.08 & 78.7 & 18.5 & 87.8 & 22.5 & 0.9 \\
\hline taurine & (tau) & 40.8 & 9.38 & 9448.8 & 2172.3 & 124.9 & 49.3 & 75.7 \\
\hline hypotaurine & (h-tau) & 1.91 & 0.65 & 442.3 & 150.5 & 2.4 & 0.7 & 184.3 \\
\hline alanine & (ala) & 2.24 & 0.83 & 518.8 & 192.2 & 387.4 & 169.5 & 1.3 \\
\hline tyrosine & (tyr) & 0.79 & 0.35 & 183.0 & 81.1 & 71.7 & 34.9 & 2.6 \\
\hline$\alpha$-aminobutyrate & (aba) & 0.16 & 0.10 & 37.1 & 23.2 & 20.3 & 8.8 & 1.8 \\
\hline tryptophane & (trp) & 0.19 & 0.11 & 44.0 & 25.5 & 71.2 & 26.1 & 0.6 \\
\hline methionine & (met) & 0.17 & 0.12 & 39.4 & 27.8 & 20.2 & 9.8 & 1.9 \\
\hline valine & (val) & 0.47 & 0.19 & 108.8 & 44.0 & 185.6 & 71.4 & 0.6 \\
\hline phenylalanine & (phe) & 1.02 & 0.64 & 236.2 & 148.2 & 84.9 & 28.5 & 2.8 \\
\hline isoleucine & (ile) & 0.52 & 0.17 & 120.4 & 39.4 & 78.4 & 35.6 & 1.5 \\
\hline leucine & (leu) & 0.83 & 0.26 & 192.2 & 60.2 & 136.5 & 86.9 & 1.4 \\
\hline ornithine & (orn) & 0.44 & 0.12 & 101.9 & 27.8 & 45.1 & 24.7 & 2.3 \\
\hline lysine & (lys) & 0.56 & 0.30 & 129.7 & 69.5 & 216.8 & 89.2 & 0.6 \\
\hline \multirow{3}{*}{$\alpha$-keto acids } & & \multicolumn{2}{|c|}{ PMN } & \multicolumn{2}{|c|}{ NCV } & \multicolumn{2}{|c|}{ Plasma } & Gradient \\
\hline & & \multicolumn{2}{|c|}{$10^{-17} \mathrm{Mol} /$ cell } & \multicolumn{2}{|c|}{ [ $\mu \mathrm{Mol} / \mathrm{l}]$} & \multicolumn{2}{|c|}{$[\mu \mathrm{Mol} / \mathrm{l}]$} & I:E \\
\hline & & \multicolumn{2}{|c|}{ mean \pm SD } & mea & SD & mea & SD & \\
\hline a-ketoglutarate & $(\mathrm{KG})$ & 1.21 & 0.31 & 30.5 & 7.8 & 6.8 & 2.3 & 4.5 \\
\hline pyruvate & (PYR) & 6.72 & 2.03 & 169.1 & 51.1 & 102.9 & 42.7 & 1.6 \\
\hline a-ketobutyrate & $(\mathrm{KB})$ & 4.07 & 1.30 & 102.4 & 32.7 & 8.7 & 2.9 & 11.8 \\
\hline a-ketoisovalerate & (KIV) & 2.27 & 1.08 & 57.1 & 27.2 & 18.1 & 7.5 & 3.2 \\
\hline a-ketoisocaproate & (KIC) & 0.36 & 0.25 & 9.1 & 6.3 & 37.5 & 11.4 & 0.2 \\
\hline p-OH-phenylpyruvate & (PPYR) & 0.29 & 0.18 & 7.3 & 4.5 & 7.6 & 4.1 & 1.0 \\
\hline$\alpha$-keto- $\beta$-methylvalerate & (KMV) & 0.80 & 0.49 & 20.1 & 12.3 & 24.2 & 8.6 & 0.8 \\
\hline
\end{tabular}




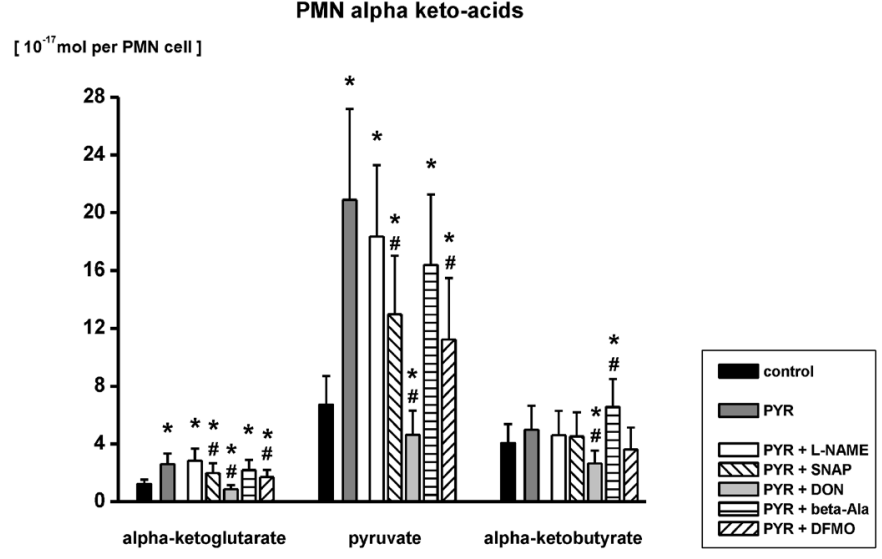

Figure 1. Effects of pyruvate $(1 \mathrm{mM}$, incubated for $120 \mathrm{~min})$ after a $10 \mathrm{~min}$ pre-incubation either with $\boldsymbol{L}$ NAME $(1 \mathrm{mM})$, SNAP $(100 \mu \mathrm{M})$, DON $(100 \mu \mathrm{M})$, beta-Ala $(10 \mathrm{mM})$ or DFMO $(1 \mathrm{mM})$ on free intracellular $\boldsymbol{\alpha}$-ketoglutarate, pyruvate and $\boldsymbol{\alpha}$-ketobutyrate concentrations in neutrophils [PMN] $\left(10^{-17}\right.$ moles per PMNcell; mean $\pm \mathrm{SD} ; \mathrm{n}=10$ ). ${ }^{*} \mathrm{p} \leq 0.05$ versus control values; ${ }^{\#} \mathrm{p} \leq 0.05$ versus pyruvate.

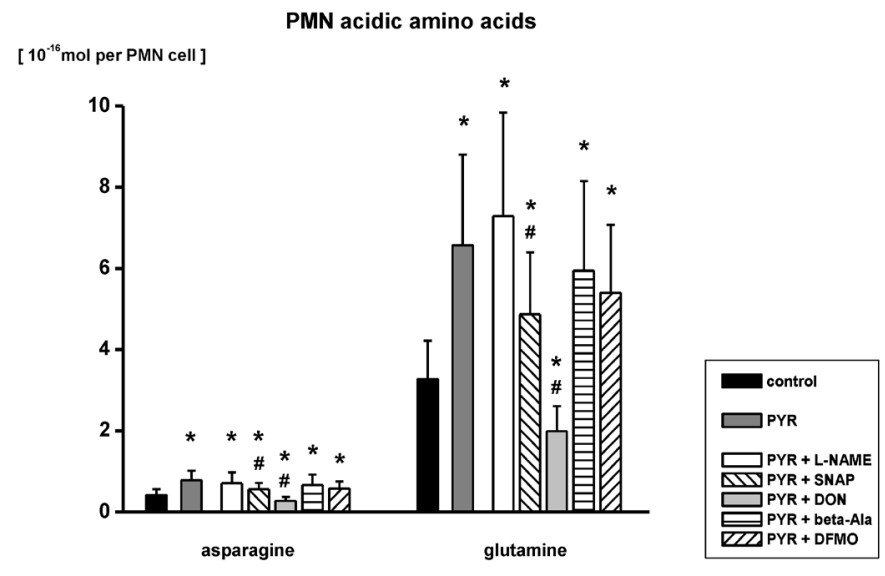

Figure 2. Effects of pyruvate $(1 \mathrm{mM}$, incubated for $120 \mathrm{~min})$ after a $10 \mathrm{~min}$ pre-incubation either with $\boldsymbol{L}$ NAME $(1 \mathrm{mM})$, SNAP $(100 \mu \mathrm{M})$, DON $(100 \mu \mathrm{M})$, beta-Ala $(10 \mathrm{mM})$ or DFMO $(1 \mathrm{mM})$ on free intracellular acidic amino acid (asparagine and glutamine) concentrations in neutrophils [PMN] $\left(10^{-16}\right.$ moles per PMNcell; mean $\pm \mathrm{SD} ; \mathrm{n}=10$ ). ${ }^{*} \mathrm{p} \leq 0.05$ versus control values; ${ }^{*} \mathrm{p} \leq 0.05$ versus pyruvate.

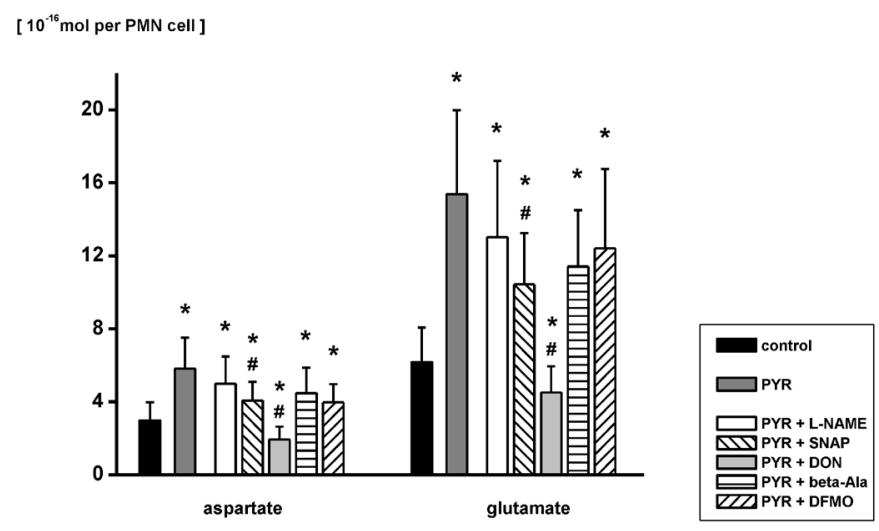

Figure 3. Effects of pyruvate $(1 \mathrm{mM}$, incubated for $120 \mathrm{~min})$ after a $10 \mathrm{~min}$ pre-incubation either with L-NAME $(1 \mathrm{mM})$, SNAP $(100 \mu \mathrm{M})$, DON $(100 \mu \mathrm{M})$, beta-Ala $(10 \mathrm{mM})$ or DFMO $(1 \mathrm{mM})$ on free intracellular acidic amino acid (aspartate and glutamate) concentrations in neutrophils [PMN] (10 ${ }^{-16}$ moles per PMN-cell; mean $\pm \mathrm{SD} ; \mathrm{n}=10$ ). ${ }^{*} \mathrm{p} \leq 0.05$ versus control values; ${ }^{\#} \mathrm{p} \leq 0.05$ versus pyruvate. 


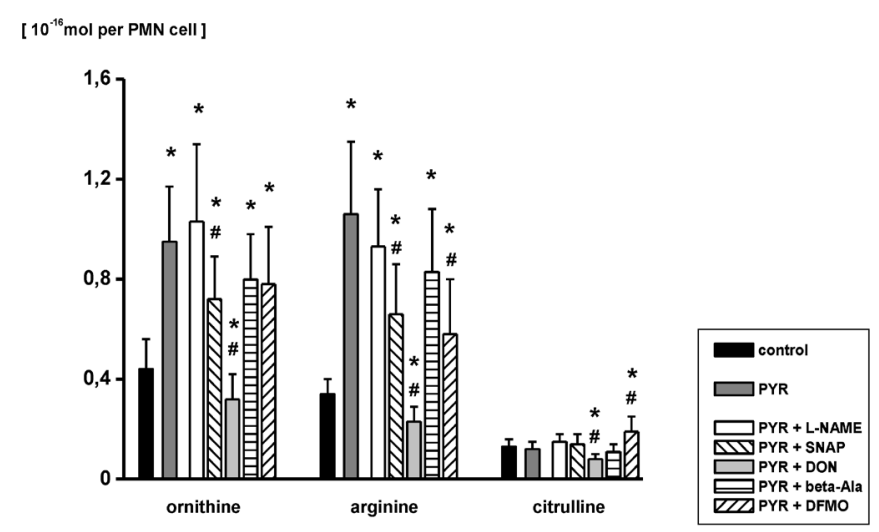

Figure 4. Effects of pyruvate $(1 \mathrm{mM}$, incubated for $120 \mathrm{~min})$ after a $10 \mathrm{~min}$ pre-incubation either with $\mathbf{L}$ NAME $(1 \mathrm{mM})$, SNAP $(100 \mu \mathrm{M})$, DON $(100 \mu \mathrm{M})$, beta-Ala $(10 \mathrm{mM})$ or DFMO $(1 \mathrm{mM})$ on free intracellular basic amino acid concentrations (ornithine, arginine, citrulline) in neutrophils [PMN] (10 ${ }^{-16}$ moles per PMN-cell; mean $\pm \mathrm{SD} ; \mathrm{n}=10$ ). ${ }^{*} \mathrm{p} \leq 0.05$ versus control values; ${ }^{\#} \mathrm{p} \leq 0.05$ versus pyruvate.

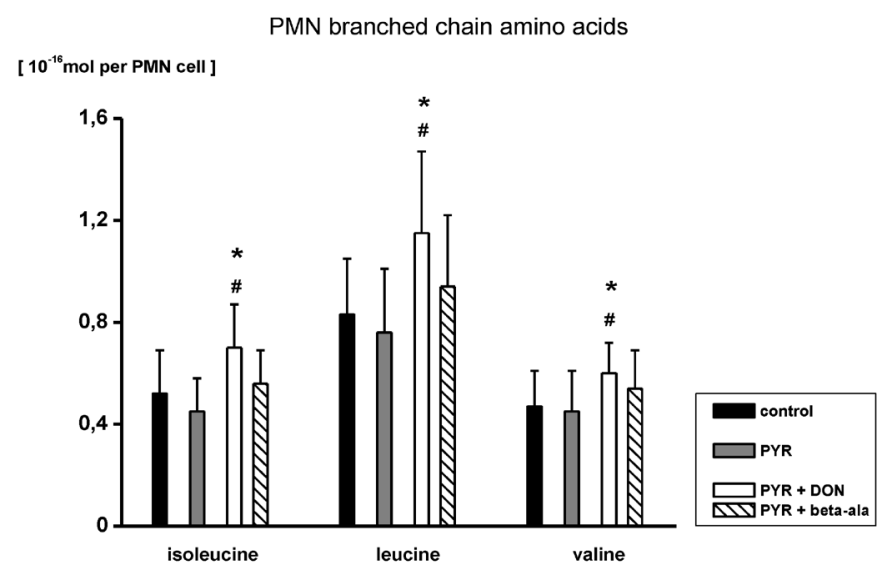

Figure 5. Effects of pyruvate (1 mM, incubated for $120 \mathrm{~min}$ ) after a $10 \mathrm{~min}$ pre-incubation either with $\mathbf{L} \mathbf{D O N}$ $(100 \mu \mathrm{M})$ or beta-Ala $(10 \mathrm{mM})$ on free intracellular branched chain amin acid concentrations (isoleucine, leucine, valine) in neutrophils [PMN] $\left(10^{-16}\right.$ moles per PMN-cell; mean $\pm \mathrm{SD}$; $\left.\mathrm{n}=10\right) .{ }^{*} \mathrm{p} \leq 0.05$ versus control values; ${ }^{\#} \mathrm{p} \leq 0.05$ versus pyruvate.

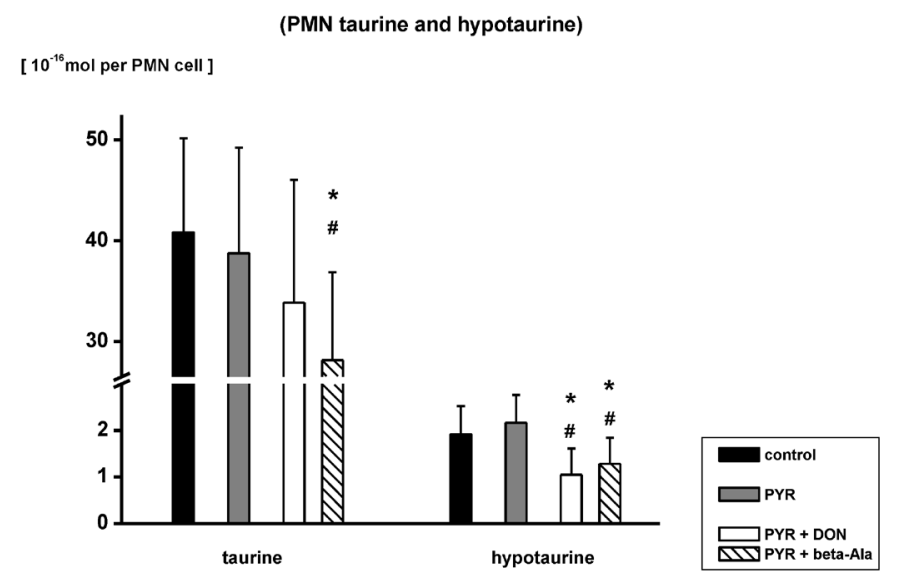

Figure 6. Effects of pyruvate ( $1 \mathrm{mM}$, incubated for $120 \mathrm{~min}$ ) after a $10 \mathrm{~min}$ pre-incubation either with $\boldsymbol{L} \mathbf{D O N}$ $(100 \mu \mathrm{M})$ or beta-Ala $(10 \mathrm{mM})$ on free intracellular taurine and hypotaurine concentrations in neutrophils [PMN] $\left(10^{-16}\right.$ moles per PMN-cell; mean $\left.\pm \mathrm{SD} ; \mathrm{n}=10\right)$. ${ }^{*} \mathrm{p} \leq 0.05$ versus control values; ${ }^{*} \mathrm{p} \leq 0.05$ versus pyruvate. 


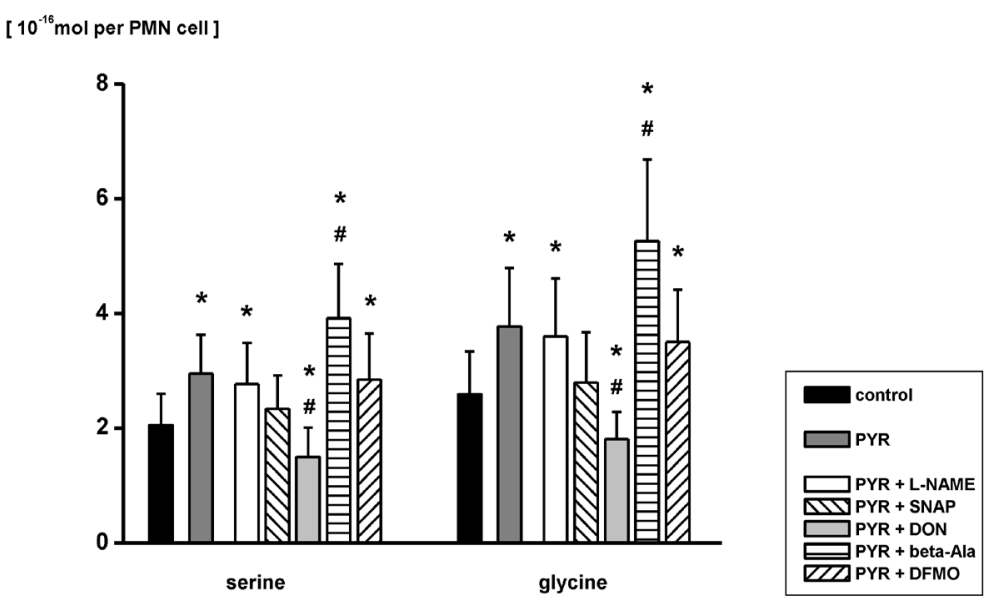

Figure 7. Effects of pyruvate $(1 \mathrm{mM}$, incubated for $120 \mathrm{~min})$ after a $10 \mathrm{~min}$ pre-incubation either with $\boldsymbol{L}$ NAME $(1 \mathrm{mM})$, SNAP $(100 \mu \mathrm{M})$, DON $(100 \mu \mathrm{M})$, beta-Ala $(10 \mathrm{mM})$ or DFMO $(1 \mathrm{mM})$ on free intracellular neutral amino acid concentrations (serine, glycine) in neutrophils [PMN] (10 ${ }^{-16}$ moles per PMN-cell; mean \pm $\mathrm{SD} ; \mathrm{n}=10) .{ }^{*} \mathrm{p} \leq 0.05$ versus control values; $\mathrm{p} \leq 0.05$ versus pyruvate.

PMN neutral amino acids

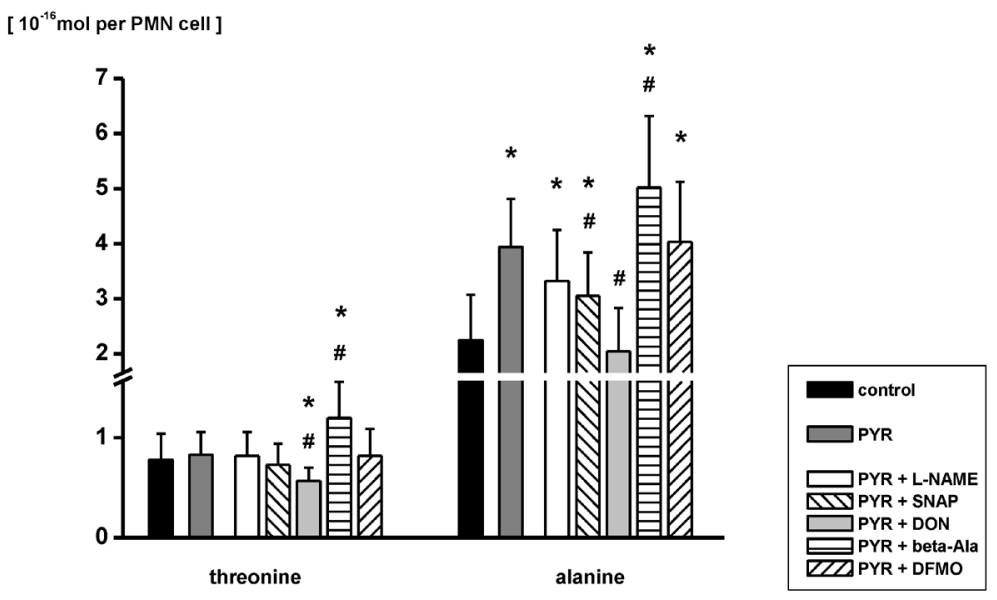

Figure 8. Effects of pyruvate $(1 \mathrm{mM}$, incubated for $120 \mathrm{~min})$ after a $10 \mathrm{~min}$ pre-incubation either with $\mathbf{L}$ NAME $(1 \mathrm{mM})$, SNAP $(100 \mu \mathrm{M})$, DON $(100 \mu \mathrm{M})$, beta-Ala $(10 \mathrm{mM})$ or DFMO $(1 \mathrm{mM})$ on free intracellular neutral amino acid concentrations (threonine, alanine) in neutrophils [PMN] $\left(10^{-16}\right.$ moles per PMN-cell; mean $\pm \mathrm{SD} ; \mathrm{n}=10$ ). ${ }^{*} \mathrm{p} \leq 0.05$ versus control values; ${ }^{*} \mathrm{p} \leq 0.05$ versus pyruvate.

Although SNAP and PYR in neutrophils, compared with PYR alone, induced significantly lower intracellular asparagine (PYR: +90\%; PYR+SNAP: +31\%), glutamine (PYR: +101\%; PYR + SNAP: +48\%), aspartate (PYR: +95\%; PYR + SNAP: +39\%), glutamate (PYR: +149\%; PYR + SNAP: +69\%) alanine and basic amino acids levels (ornithine: PYR: +115\%; PYR+SNAP: +63\%, arginine: PYR: +211\%; PYR + SNAP: +94\%, citrulline: PYR: $-8 \%$; PYR+SNAP: +7\%), the results were nevertheless still significantly above the control findings. For serine (PYR: +43\%; PYR + SNAP: +14\%), glycine (PYR: +47\%; PYR + SNAP: +8\%) and threonine (PYR: $+6 \%$; PYR + SNAP: $-7 \%$ ) as well, SNAP induced a concentration decrease compared to PYR application alone. Nevertheless this reduction remained more or less within the range of control values.

DON produced a very clear reduction in important primary amino acids. Although the contents of cellular alanine (PYR: +75\%; PYR + DON: -9\%) were decreased significantly compared to PYR singular application, they were still within control ranges. Regarding the granulocytic asparagine (PYR+DON: $-34 \%$ ), aspartate (PYR + SNAP: -29\%), glutamine (PYR + SNAP: -41\%), glutamate (PYR + SNAP: -27\%), branch-chain amino acid levels (isoleucine: PYR: -13\%; PYR + DON: +34\%, leucine: PYR: -8\%; PYR + DON: +39\%, valine: PYR: $-4 \%$; PYR + DON: +30\%), hypotaurine (but not taurine, PYR: +13\%; PYR + DON: $-45 \%$ ), neutral 
amino acids (serine: PYR + DON: $-27 \%$, glycine: PYR + DON: +31\%, threoni-ne: PYR + DON: $-25 \%$ ) as well as basic amino acid levels (ornithine: PYR + DON: $-28 \%$, arginine: PYR + DON: $-33 \%$, citrulline: PYR + DON: $-38 \%$ ), significant intracellular concentration changes were observed.

$\beta$-Ala in combination with PYR induced significant reductions in granulocytic hypotaurine (PYR $+\beta$-Ala: $-39 \%$ ) and taurine (PYR $+\beta$-Ala: $-31 \%$ ). In addition, $\beta$-Ala also exerted additive effects on the content of neutral amino acids (serine: PYR $+\beta$-Ala: $+91 \%$, glycine: PYR $+\beta$-Ala: $+104 \%$, threonine: PYR $+\beta$-Ala: $+53 \%$, alanine: PYR $+\beta$-Ala: $+124 \%)$.

DFMO added to PYR (similar to L-NAME) induced a decrease in intragranulocytic arginine (PYR + DFMO versus PYR: $-45 \%$ ) and increase in citrulline (PYR + DFMO versus PYR: $+58 \%$ ) that exceeded the effects of singular PYR application. The slight decrease of ornithine was not statistically significant (DFMO added to PYR).

\subsection{Effects of PYR Combined with L-NAME, SNAP, DON, $\beta$-Ala or DFMO on Neutrophil Oxidative Response and Myeloperoxidase Activity (Figure 9)}

PYR led to an increased formation of superoxide anions and hydrogen peroxide and combined with L-NAME additional significant increases were observed $\left(\mathrm{O}_{2}^{-}\right.$: PYR: $+155 \%$; PYR + L-NAME versus PYR: $+87 \%$; $\mathrm{H}_{2} \mathrm{O}_{2}$ : PYR: +123\%; PYR + L-NAME versus PYR: $+128 \%)$. The activity of myeloperoxidase released by neutrophils on the other hand was not influenced by L-NAME application (PYR: $+164 \%$ ).

In combination with PYR, SNAP and DON induced decreases in superoxide anion and hydrogen peroxide generation that were raised by PYR on its own, but DON alone however led to a reduction below control values $\left(\mathrm{O}_{2}^{-}\right.$: PYR + DON: $-31 \% ; \mathrm{H}_{2} \mathrm{O}_{2}$ : PYR + DON: $\left.-36 \%\right)$. The "nutritively"-induced increase in myeloperoxidase activity was also significantly inhibited by SNAP and DON but the results revealed no differences from control findings.

$\beta$-alanine in combination with PYR led to a significant increase in granulocytic superoxide anion production. With PYR there were additive effects exceeding effects of singular incubation (PYR $+\beta$-Ala versus PYR: $+39 \%)$. Similar effects were seen with hydrogen peroxide formation, where there were also further significant increases in the measured values (PYR $+\beta$-Ala versus PYR: $+67 \%$ ). Regarding the myeloperoxidase activity, the combination of $\beta$-alanine and PYR did not lead to significant effects.

DFMO also produced an inhibition of superoxide anion and hydrogen peroxide formation. The findings obtained here for PYR were completely above the respective control values $\left(\mathrm{O}_{2}^{-}\right.$: PYR+DFMO versus PYR: $-41 \%$ or versus control: $+49 \% ; \mathrm{H}_{2} \mathrm{O}_{2}$ : PYR + DFMO versus PYR: $-28 \%$ or versus control: $\left.+62 \%\right)$.

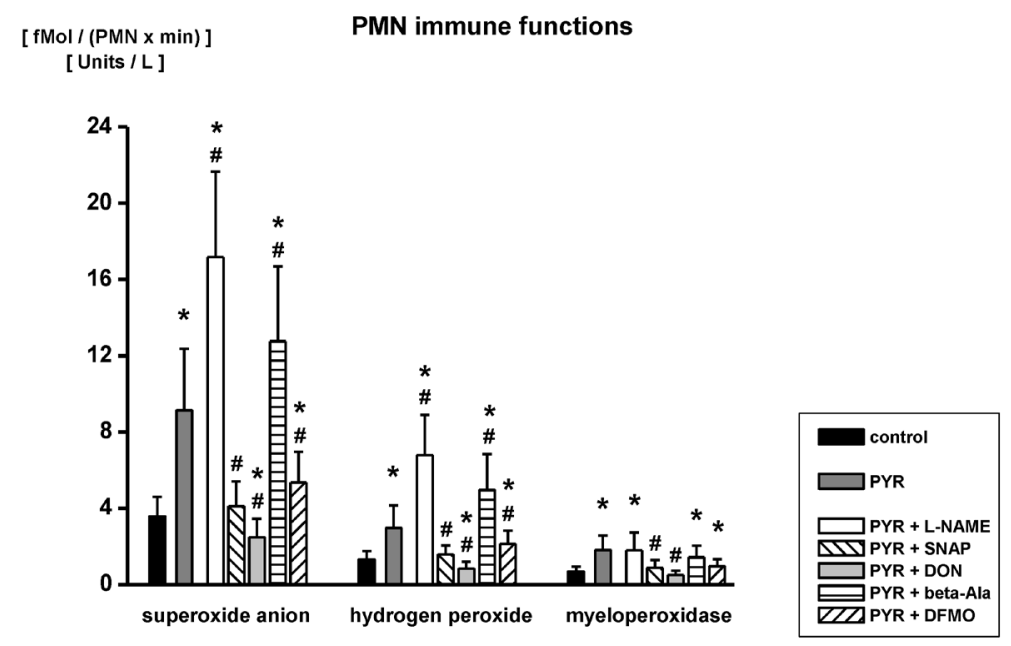

Figure 9. Effects of pyruvate $(1 \mathrm{mM}$, incubated for $120 \mathrm{~min})$ after a $10 \mathrm{~min}$ pre-incubation either with $\boldsymbol{L}$ NAME $(1 \mathrm{mM})$, SNAP $(100 \mu \mathrm{M})$, DON $(100 \mu \mathrm{M})$, beta-Ala $(10 \mathrm{mM})$ or DFMO $(1 \mathrm{mM})$ on PMN superoxide anion production $\left[\mathrm{O}_{2}^{-} ; \mathrm{fmol} /(\mathrm{PMN} \cdot \mathrm{min})^{-1}\right]$, hydrogen peroxide formation $\left[\mathrm{H}_{2} \mathrm{O}_{2} ; \mathrm{fmol} /(\mathrm{PMN} \cdot \mathrm{min})^{-1}\right]$ and myeloperoxidase activity [MPO; units/l supernatant]; (mean $\pm \mathrm{SD} ; \mathrm{n}=10$ ). ${ }^{*} \mathrm{p} \leq 0.05$ versus control values; ${ }^{*} \mathrm{p}$ $\leq 0.05$ versus pyruvate. 


\section{Discussion}

It is broadly accepted scientific consensus that not only amino acids (i.e. taurine, glutamine, glutamate, alanine, arginine or ornithine) but also $\alpha$-keto acids (i.e. $\alpha$-ketoglutarate, pyruvate) appear to have significant metabolic and nutritional functions in leukocytes. More than that, the composition of free intracellular amino acid and $\alpha$-ketoacid pools in body cells do not seem to be arbitrary in any direction, but may rather represent a relevant metabolic correlate, a quasi-immunonutritional metabolic fingerprint, for the underlying functions [24]-[26]. It is thus not surprising that scientific interest here also focused on the possibility to use selected amino- or ketoacids as part of a "standard" diet therapeutically [27]-[29]. However, many questions are unfortunately here still unclear. For instance, little evidence is available regarding the question what precise pathophysiological mechanisms are involved in the effects of pyruvate on PMN substrate and energy supply or their immune functions.

\subsection{Pyruvate Induced Effects: Is There an Impact by Adding a Glutamine-Analogue?}

DON, a wide ranging inhibitor of all glutamine dependent intracellular effects, induced a clear reduction in intragranulocytic glutamine, but also contributed to important changes in pyruvate, $\alpha$-ketoglutarate or $\alpha$-ketobutyrate concentrations. Other important amino acids such as glutamate, aspartate or basic, branched chain of neutral amino acids were also significantly changed. Referring to previous research, it seems clear: the enzymatic constellation required for a direct or indirect pyruvate metabolization and synthesis via glutamine-dependent intracellular pathways is certainly present in neutrophils as it is in other leukocytes (i.e. reversible transaminations involving the release of $\mathrm{NH}_{3}$, the metabolization via phosphate-dependent glutaminase which in leukocytes can be activated by proinflammatory mediators and catecholamines, the conversion of malate to oxalacetate by the $\mathrm{NADP}^{+}$-dependent malate dehydrogenase or oxidative glutamate deamination to form ammonium ions, which are excreted as urea or also a $\alpha$-ketoglutarate synthesis from oxaloacetate and glutamate). For example $\alpha$-ketoglutarate, the carbon backbone arising from pyruvate transmission or glutamine oxidation, can immediately be fed into the tricarboxylic acid cycle and through that it is indirectly available to the enzymes of the urea cycle, for example in the form of aspartate via oxaloacetate [1]-[3] [30] [31]. So it is not surprising that $\alpha$-ketoglutarate, for example incubated in neutrophils is able to increase cellular substrate storages and immune functions [6] [32].

Moreover, as shown here, the indirect participation of pyruvate in glutamine metabolisation can also determine the formation of arginine and ornithine (i.e. catalysed by the enzymes pyrroline-5-carboxylate synthase, ornithine aminotransferase, ornithine carbamyltransferase, or carbamylphosphate synthase) or the synthesis of -NO which is required for neutrophil activation [33] [34]. And indeed, a growing consensus exists regarding a so-called "pyruvate-glutamate-ketoglutarate-arginine-ornithine-system", which especially in leukocytes, appears to hold a key role as an "intracellular turntable for amino nitrogen metabolisation" [32] [35]. The advantages to the cell seems obvious: the possibility of a rapid transition into other essential cell substrates (i.e. acetyl-CoA). Interestingly, since the enzyme fructose-1,6-bisphosphatase is also found in neutrophils, pyruvate is also seen as an indirect precursor of the de novo synthesis of glucose from pyruvate [36].

Nevertheless, it is mostly concurred that only a minuscule proportion of the carbon, arising from pyruvate incubation in neutrophils, is completely oxidized into carbon dioxide (30\%). The underlying thesis is, that in anabolic situations not only a raised pyruvate uptake, but also a substantial amount of de novo intracellular pyruvate as well as pyruvate-dependent metabolite synthesis occurs [37]-[39]. And indeed, our results seem to confirm this hypothesis, because following pyruvate incubation increased glutamate, glutamine, aspartate (asparagine), alanine, $\alpha$-ketotglutarate but also to serine and glycine concentrations have intracellularly been found (i.e. via serine- and glycine-hydroxymethyltransferase). This final detail, in particular, is of special importance for neutrophils or other leukocytes, because they must suddenly increase their metabolism and substrate turnover in response to a stimulus, and that only works if the substrate storages are filled. And the advantage of high intragranulocytic pyruvate, glutamine, glutamate, alanine or aspartate levels in the cell is obvious: neutrophils, particularly in the context of an increased demand or those which are isolated from a direct nutrient supply (i.e. in inflammation or tumour areas which per se are substrate depleted areas), can rapidly fall back on relevant metabolic precursors, required for the synthesis of immune-functional enzymes, proteins, amino sugars, RNA and DNA or glutathione. Therefore, these cells act both, as immunological fighting machines as well as nutrient transporters [40] [41].

On the base of our findings and other results available, we therefore believe, that pyruvate plays a crucial 
function as an immuonutritional energy supplier for leukocyte cells. And in fact, the energy output is high, because the transition of one molecule of pyruvate via the TCA cycle, together with oxidative phosphorylation, produces 15 molecules of ATP as well as NADPH, which can for example be further metabolized by membranous NADPH oxidase (nicotinamide adenine dinucleotide phosphate-oxidase) as well [9] [10] [42]-[44]. Under physiological conditions, the complex is inactive and only activated during respiratory burst. But after activating, this enzyme system produces enormous amounts of superoxide anion, a reactive free-radical, by transferring electrons from NADPH inside the cubicle across the membrane and coupling these with molecular oxygen. Superoxide anion can be produced in phagosomes, which contain ingested bacteria and fungi, or can be utilized as an assault weapon for external enemies by inactivation of critical metabolic enzymes, initiation of lipid peroxidation, or liberation of redox-active iron, which permits the generation of indiscriminate oxidants such as the hydroxyl radical. In a phagosome as well as in an extracellular matrix, superoxide can spontaneously form hydrogen peroxide or hypochlorous acid, also recognised as "reactive oxygen species" (ROS) [30] [45]. And indeed, these weapons are disastrous. As studies impressively demonstrated: superoxide is able to kill bacteria directly, especially when the virulence of the pathogen is dramatically attenuated (i.e. by superoxide dismutase deficiency) [46] [47]. Here in this study, the inhibition of specific glutamine-dependent pathways were one important constituent in the significant turnaround of the pyruvate-induced alterations in the immune parameters examined. We thus strongly suggest that in neutrophils a) pyruvate appears to run a relevant immunonutrional role and b) pyruvate- and glutamine-dependent pathways seem connected to each other.

\subsection{Pyruvate Induced Effects: Is There an Impact by Adding an Inhibitor of •NO-Synthase, an - NO Donor or an Inhibitor of Ornithine-Decarboxylase?}

Significant alterations of the pyruvate induced effects on the parameters observed here was also followed after a pre-incubation with an $\bullet \mathrm{NO}$ donor which artificially-induced an extracellular $\bullet \mathrm{NO}$ increase over the micromolar concentration range. For pyruvate itself, but also for $\alpha$-ketoglutarate glutamine, glutamate, ornithine, arginine or citrulline a significant reduction of the pyruvate-induced effects could be noticed, although the drop did not decrease under the initial points. Parallel results were likewise found for superoxide anion and hydrogen peroxide generation.

Regarding possible causes we believe-in line with other investigators - that it is probably a combination of different mechanisms may here be responsible for the interesting effects (i.e. the inhibition of specific pyruvate-dependent intracellular metabolic functions, a $\bullet$ NO-mediated transmembranous amino or $\alpha$-keto acid loss, a -NO-mediated influencing by enzymes of the citric acid cycle [i.e. cis-aconitase (, etc.), but the real biochemical background is yet not entirely realised [30] [45].

But in terms of the investigated immune parameters, a potential explanation may be found: in neutrophils, -NO seems directly to be involved in different mechanisms which controls the vital balance between oxygen radical metabolites in the regulation of inflammatory processes. For example, via inhibition of the NADPH oxidase complex, an inhibition of $\bullet \mathrm{NO}$ synthase, an $\bullet$ NO-mediated influencing of enzymes of the respiratory chain or via an $\bullet$ NO concentration-dependent inhibition of ribonucleotide reductase [DNA synthesis, ADP-ribosyltransferase or glyceraldehyde-3-phosphate-dehydrogenase (glycolysis and gluconeogenesis)]. And the profit for the cell seems here to be clear: these mechanisms help to protect against the danger of self-destruction and the growth of an unruly, self-reinforcing immune response [20] [48]-[50]. And this, in the end, protects all cells of the inflammatory matrix against uncontrolled tissue destruction [51].

Moreover, the outcomes of our study also showed, that pre-incubation with an ornithine decarboxylase inhibitor, led to a modulation of the pyruvate induced effects, as well. We therefore must assume, that in neutrophils inhibition of intracellular ornithine metabolism also seems to possess a considerable influence on intracellular amino- and keto acid metabolism as well as essential immune functions [52]. The most convincing hypothesis to explain these findings is, that in neutrophils and other leukocytes ornithine seems to be employed as a substrate for polyamine synthesis, which are essential metabolic components for virtually all cell types with high cell division rates and stimulable metabolic activities [53] [54]. It is believed that polyamines may have specific roles in cellular protein biosynthesis, growth, proliferation and apoptosis processes, for instance through the stabilization of cellular DNA and RNA through the binding of spermine and spermidine. And equally in other leukocytes, the need for polyamines is covered by both, transport from the extracellular matrix (here the capacity in leukocytes is low) as well as synthesis (spermine synthesis after enzymatic decarboxylation of ornithine by ornithine-decarboxylase) [55]-[59]. Nevertheless, their precise metabolic functions in human neutrophils are still 
virtually undiscovered.

Concerning free amino- and $\alpha$-keto acid pools as well as myeloperoxidase activity in neutrophils, an inhibitor of •NO-synthase (i.e. L-NAME) induced no further effects, but led to significant increases in the formation of superoxide anions and hydrogen peroxide. For this reason we think that metabolic alterations are probably not the main cause of these effects. With regard to possible causes, the following hypotheses are currently discussed: a potential influence on the transmembranous arginine transport (accompanied by a decrease in intracellular arginine content) and/or •NO-synthase independent mechanisms (i.e. through a direct reaction of arginine with $\mathrm{H}_{2} \mathrm{O}_{2}$, etc.). But in the authors' opinion, it is also conceivable that these findings, again, only indicate a critical balance and negative feedback mechanism to balance oxygen radical metabolites and $\bullet$ NO-production as a protective against self-destruction [60]-[62]. This theory, indeed, could further explain some other confusing biphasic findings previously found: while low $\bullet \mathrm{NO}$ and peroxynitrite concentrations were able to induce PMNactivation (i.e. detectable by raising production of superoxide anions and hydrogen peroxide), high $\bullet$ NO concentrations and the follow-on product, peroxynitrite or other reactive nitrogen metabolites-which can no longer be scavenged by the oxygen radicals in the extracellular matrix-reverse this process [63]. Interestingly, these effects seem to be multifactorial here (i.e. perhaps via a $\bullet$ NO-dose-dependent inhibition of reduction of superoxide anion generation and NADPH oxidase complex or $\bullet$ NO synthase $\beta$-glucuronidase secretion and leukotriene formation) [47]. On the other hand, nevertheless, an inhibition of $\bullet$ NO-synthesis combined with an immunonutrition of neutrophils (i.e. Using pyruvate) leads to a substantial growth of oxygen free radicals that cannot be further metabolized by $\bullet$ NO [64] [65]. Our assumption is therefore: the L-NAME-induced further changes in superoxide anion and hydrogen peroxide formation may not primarily represent an activation of neutrophils, but seems simply to reflect a reduced conversion of $\mathrm{NO}$ and oxygen radicals to further metabolites (i.e. peroxynitrite, etc.) [48] [60] [63].

\subsection{Pyruvate Induced Effects: Is There an Impact by Adding a Taurine Transport Antagonist?}

The results of this study additionally showed, that pyruvate had no influence on the taurine and hypotaurine content, only the loudness of the taurine-hypotaurine-amino acid transport system (i.e. following pre-incubation with $\beta$-alanine) led to significant decreases in intragranulocytic taurine and hypotaurine concentrations (interstingly, very similar effects were observed after incubation with DON) as well as resulted to parallel increases in intracellular neutral amino acids and $\alpha$-ketobutyrate (which are metabolically related to threonine). Touching on the PMN immune parameters observed here, $\beta$-alanine, in combination with pyruvate, led to further additive effects in granulocytic superoxide anion production and hydrogen peroxide formation, but simply possessed no influence on the activity of myeloperoxidase.

Adverting to the interesting new results we believe that a modulation of relevant taurine-dependent transmembraneous transport mechanisms are the most likely explanation for the changes in intragranulocyte taurine concentrations. But only a simple homeostatic response to modifications in intragranulocytic amino- and $\alpha$-keto acid homeostasis or metabolism can also not be excludedt [5] [66]. These novel results, again, support the hypothesis that compositional modifications in neutrophil taurine concentrations appear to affect the composition of the intragranulocytic amino- and $\alpha$-keto acid pools but certainly influences the magnitude and the character of their immune response. Not least the exceptional high taurine concentrations in neutrophils compared with other leukocytes, seem to support this hypothesis [67]-[69].

\section{Conclusion}

In summary, the interesting results presented here showed, that any damage of specific pathways or mechanisms, which seem directly or indirectly connected to pyruvate conversion-dependent cellular tasks, led to significant immunonutritional alterations in the affected PMN-cells. We thus believe that pyruvate, irrespective of which exact biochemical mechanisms were involved, may satisfy the substantial metabolic demands for a potent intracellular nutrient. With respect to a clinical therapy, but there are problems: pyruvate is relatively unstable in aqueous solution and the hunt for a possible alternative here currently led, for example, to the development of ethyl pyruvate, which is a simple aliphatic ester derived from pyruvate, but as well contributed to the invention of stable aqueous solutions containing sodium pyruvate using $\mathrm{H}^{+}(\mathrm{pH} 3.0-6.0)$ or additional $\mathrm{Na}^{+}(20 \mathrm{mM})$ [22] [70]-[72]. And in fact, the first results seem to be promising: treatment with pyruvate or ethyl pyruvate has 
shown to improve survival and/or ameliorate organ dysfunction in a wide variety of pre-clinical models or clinical trials of acute illnesses such as severe sepsis, acute pancreatitis, respiratory distress syndromes, stroke, cancer or in patients undergoing cardiac surgery [18] [35] [70]-[79]. Furthermore, the therapy using pyruvate or pyruvate-related compounds was also shown to be safe, but, nevertheless, in most studies it failed to improve outcome. So, we believe that the following consideration could play an important function here: concerning any immunonutritional therapy using pyruvate or derivatives, one should bear in mind that any maintaining or increasing of a granulocytic metabolic function or any improvement of granulocyte specific immunological weapons must not inevitably contribute to a clinical improvement of the disease outcome; and especially not in critically ill patients. This certainly surprising thesis can partially be explained by the pathophysiological problem that arises when immunonutritionally strengthened neutrophils migrate into an inflammatory nutrient depleted, and thereby potentially weakened, extravascular matrix. Here, in that location is indeed a substantial hazard of an additional granulocyte-mediated cell and tissue trauma. Immunonutrition using pyruvate or pyruvate derived chemical compounds (e.g. ethyl-pyruvate), may therefore possess a dual-headed nature, best symbolised by “Janus", the god of beginnings and transitions, gates, doorways, endings and time [32] [40] [80]-[82].

In our view, more research activity seems to be necessary and useful to conclusively clarify the important therapeutic question: Is a therapy, using pyruvate or pyruvate derived chemical compounds, really secure and beneficial for our patients?

\section{References}

[1] Frei, J., Aellig, A. and Nessi, P. (1975) Enzyme System and Coenzymes Involved in the Energy Metabolism of Leukocytes. Function and Metabolism of Polymorphonuclear Neutrophils. Annales de Biologie Clinique (Paris), 33, 459-464.

[2] Frei, J. and Jemelin, M. (1972) Metabolism of Leukocytes in Health and Disease. Introduction, an Approach to Direct Metabolic Studies in Man. Enzyme, 13, 3-6.

[3] Frei, J., Maillard, M., Markert, M. and Aellig, M. (1980) Energetic Metabolism of Leukocytes. XI. Presence and Function of Creatine Kinase in Leukocytes. Enzyme, 25, 258-264.

[4] Jambunathan, P. and Zhang, K. (2014) Novel Pathways and Products from 2-Keto Acids. Current Opinion in Biotechnology, 29c, 1-7. http://dx.doi.org/10.1016/j.copbio.2014.01.008

[5] Mühling, J., Nickolaus, K.A., Halabi, M., Fuchs, M. and Krüll, M. (2005) Alterations in Neutrophil (PMN) Free Intracellular Alpha-Keto Acid Profiles and Immune Functions Induced by L-Alanyl-L-Glutamine, Arginine or Taurine. Amino Acids, 29, 289-300. http://dx.doi.org/10.1007/s00726-005-0223-8

[6] Mühling, J., Tussing, F., Nickolaus, K.A., Matejec, R. and Henrich, M. (2010) Effects of Alpha-Ketoglutarate on Neutrophil Intracellular Amino and Alpha-Keto Acid Profiles and ROS Production. Amino Acids, 38, 167-177. http://dx.doi.org/10.1007/s00726-008-0224-5

[7] Markert, M. and Frei, J. (1978) Energy Metabolism of Polymorphonuclear Neutrophils and Phagocytosis (Proceedings). Annales de Biologie Clinique (Paris), 36, 201-204.

[8] Stjernholm, R.L., Burns, C.P. and Hohnadel, J.H. (1972) Carbohydrate Metabolism by Leukocytes. Enzyme, 13, 7-31.

[9] Stjernholm, R.L., Dimitrov, N.V. and Pijanowski, L.J. (1969) Carbohydrate Metabolism in Leukocytes. IX. Citric Acid Cycle Activity in Human Neutrophils. Journal of the Reticuloendothelial Society, 6, 194-201.

[10] Sznajd, J., Malkiewicz-Wasowicz, B., Naskalski, J. and Lisiewicz, J. (1972) Biochemistry of Normal and Leukemic Leukocytes. X. Energy and Carbohydrate Metabolism of Neutrophilic Granulocytes. Przegl Lek, 29, 634-640.

[11] He, G., Jiang, Y., Zhang, B. and Wu, G. (2014) The Effect of HIF-1 $\alpha$ on Glucose Metabolism, Growth and Apoptosis of Pancreatic Cancerous Cells. Asia Pacific Journal of Clinical Nutrition, 23, 174-180.

[12] Hou, Y.Q., Wang, L., Ding, B., Liu, Y., Zhu, H., Liu, J., Li, Y.T., Kang, P., Yin, Y.L. and Wu, G.Y. (2011) AlphaKetoglutarate and Intestinal Function. Frontiers in Bioscience (Landmark Ed), 16, 1186-1196. http://dx.doi.org/10.2741/3783

[13] Tang, D., Kang, R., Zeh III, H.J. and Lotze, M.T. (2011) High-Mobility Group Box 1, Oxidative Stress, and Disease. Antioxidants \& Redox Signaling, 14, 1315-1335. http://dx.doi.org/10.1089/ars.2010.3356

[14] Fuchs, M., Engel, J., Campos, M., Matejec, R., Henrich, M., Harbach, H., et al. (2009) Intracellular Alpha-Keto Acid Quantification by Fluorescence-HPLC. Amino Acids, 36, 1-11. http://dx.doi.org/10.1007/s00726-008-0033-x

[15] Roth, E. (2008) Nonnutritive Effects of Glutamine. Journal of Nutrition, 138, 2025S-2031S.

[16] Cai, B., Deitch, E.A. and Ulloa, L. (2010) Novel Insights for Systemic Inflammation in Sepsis and Hemorrhage. Mediators of Inflammation, 2010, Article ID: 642462. 
[17] Deng, Z.H., Ti, D.D., Xue, H., Lin, J., Wang, L.H. and Yan, G.T. (2009) Effects of Ethyl Pyruvate on Injuries of Sepsis in Mice. Zhongguo Wei Zhong Bing Ji Jiu Yi Xue, 21, 460-462.

[18] Fink, M.P. (2008) Ethyl Pyruvate. Current Opinion in Anaesthesiology, 21, 160-167. http://dx.doi.org/10.1097/ACO.0b013e3282f63c2e

[19] Jacobs, C.C., Holcombe, S.J., Cook, V.L., Gandy, J.C., Hauptman, J.G. and Sordillo, L.M. (2013) Ethyl Pyruvate Diminishes the Inflammatory Response to Lipopolysaccharide Infusion in Horses. Equine Veterinary Journal, 45, 333339. http://dx.doi.org/10.1111/j.2042-3306.2012.00634.X

[20] Kung, C.W., Lee, Y.M., Cheng, P.Y., Peng, Y.J. and Yen, M.H. (2011) Ethyl Pyruvate Reduces Acute Lung Injury via Regulation of iNOS and HO-1 Expression in Endotoxemic Rats. Journal of Surgical Research, 167, e323-e331. http://dx.doi.org/10.1016/j.jss.2011.01.006

[21] Onur, E., Akalin, B., Memisoglu, K., Karip, A.B., Aydin, M.T., Altun, H. and Ekci, B. (2012) Ethyl Pyruvate Improves Healing of Colonic Anastomosis in a Rat Model of Peritonitis. Surgical Innovation, 19, 394-398. http://dx.doi.org/10.1177/1553350611432158

[22] Zhou, F. (2009) Stable Aqueous Solution Containing Sodium Pyruvate, and the Preparation Thereof. IPC8 Class: AA61K3314FI, USPC Class: 424677.

[23] Mathioudakis, D., Engel, J., Welters, I.D., Dehne, M.G., Matejec, R., Harbach, H., et al. (2011) Pyruvate: Immunonutritional Effects on Neutrophil Intracellular Amino or Alpha-Keto Acid Profiles and Reactive Oxygen Species Production. Amino Acids, 40, 1077-1090. http://dx.doi.org/10.1007/s00726-010-0731-z

[24] Mühling, J., Fuchs, M., Dehne, M.G., Sablotzki, A., Menges, T., Weber, B. and Hempelmann, G. (1999) Quantitative Determination of Free Intracellular Amino Acids in Single Human Polymorphonuclear Leucocytes: Recent Developments in Sample Preparation and High-Performance Liquid Chromatography. Journal of Chromatography B: Biomedical Sciences and Applications, 728, 157-166. http://dx.doi.org/10.1016/S0378-4347(99)00114-0

[25] Mühling, J., Fuchs, M., Campos, M.E., Gonter, J., Engel, J.M., Sablotzki, A., et al. (2003) Quantitative Determination of Free Intracellular Alpha-Keto Acids in Neutrophils. Journal of Chromatography B, 789, 383-392. http://dx.doi.org/10.1016/S1570-0232(03)00163-6

[26] Mühling, J., Fuchs, M., Fleck, C., Sablotzki, A., Krüll, M., Dehne, M.G., Gonter, J., Weiss, S., Engel, J. and Hempelmann, G. (2002) Effects of Arginine, L-alanyl-L-glutamine or Taurine on Neutrophil (PMN) Free Amino Acid Profiles and Immune Functions in Vitro. Amino Acids, 22, 39-53. http://dx.doi.org/10.1007/s726-002-8200-9

[27] Abunnaja, S., Cuviello, A. and Sanchez, J.A. (2013) Enteral and Parenteral Nutrition in the Perioperative Period: State of the Art. Nutrients, 5, 608-623. http://dx.doi.org/10.3390/nu5020608

[28] Pierre, J.F., Heneghan, A.F., Lawson, C.M., Wischmeyer, P.E., Kozar, R.A. and Kudsk, K.A. (2013) Pharmaconutrition Review, Physiological Mechanisms. JPEN Journal of Parenteral and Enteral Nutrition, 37, 51s-65s. http://dx.doi.org/10.1177/0148607113493326

[29] Weimann, A. (2013) Immunonutrition in Intensive Care Medicine. Medizinische Klinik, Intensivmedizin und Notfallmedizin, 108, 85-94; Quiz 95.

[30] Gabrilovich, D.I. (1999) The Neutrophils, New Outlook for Old Cells. Imperial College Press, London.

[31] Mühling, J., Fuchs, M., Campos, M., Gonter, J., Sablotzki, A., Engel, J., et al. (2004) Effects of Ornithine on Neutrophil (PMN) Free Amino Acid and Alpha-Keto Acid Profiles and Immune Functions in Vitro. Amino Acids, 27, 313-319. http://dx.doi.org/10.1007/s00726-004-0126-0

[32] Mates, J.M., Segura, J.A., Martin-Rufian, M., Campos-Sandoval, J.A., Alonso, F.J. and Marquez, J. (2013) Glutaminase Isoenzymes as Key Regulators in Metabolic and Oxidative Stress against Cancer. Current Molecular Medicine, 13, 514-534. http://dx.doi.org/10.2174/1566524011313040005

[33] Moinard, C., Caldefie, F., Walrand, S., Tridon, A., Chassagne, J., Vasson, M.P. and Cynober, L. (2002) Effects of Ornithine 2-Oxoglutarate on Neutrophils in Stressed Rats: Evidence for the Involvement of Nitric Oxide and Polyamines. Clinical Science, 102, 287-295. http://dx.doi.org/10.1042/CS20010162

[34] Moinard, C., Caldefie-Chezet, F., Walrand, S., Vasson, M.P. and Cynober, L. (2002) Evidence that Glutamine Modulates Respiratory Burst in Stressed Rat Polymorphonuclear Cells through Its Metabolism into Arginine. British Journal of Nutrition, 88, 689-695. http://dx.doi.org/10.1079/BJN2002724

[35] Demaria, S., Pikarsky, E., Karin, M., Coussens, L.M., Chen, Y.C., et al. (2010) Cancer and Inflammation, Promise for Biologic Therapy. Journal of Immunotherapy, 33, 335-351. http://dx.doi.org/10.1097/CJI.0b013e3181d32e74

[36] Sybirna, N., Dziewulska-Szwajkowska, D., Barska, M. and Dzugaj, A. (2006) Mononuclear and Polymorphonuclear Leukocytes Show Increased Fructose-1,6-Bisphosphatase Activity in Patients with Type 1 Diabetes Mellitus. Cell Biology International, 30, 624-630. http://dx.doi.org/10.1016/j.cellbi.2006.03.008

[37] Castell, L., Vance, C., Abbott, R., Marquez, J. and Eggleton, P. (2004) Granule Localization of Glutaminase in Human Neutrophils and the Consequence of Glutamine Utilization for Neutrophil Activity. Journal of Biological Chemistry, 
279, 13305-13310. http://dx.doi.org/10.1074/jbc.M309520200

[38] Newsholme, P., Curi, R., Pithon Curi, T.C., Murphy, C.J., Garcia, C. and Pires de Melo, M. (1999) Glutamine Metabolism by Lymphocytes, Macrophages, and Neutrophils: Its Importance in Health and Disease. Journal of Nutritional Biochemistry, 10, 316-324. http://dx.doi.org/10.1016/S0955-2863(99)00022-4

[39] Pithon-Curi, T.C., De Melo, M.P. and Curi, R. (2004) Glucose and Glutamine Utilization by Rat Lymphocytes, Monocytes and Neutrophils in Culture: A Comparative Study. Cell Biochemistry and Function, 22, 321-326. http://dx.doi.org/10.1002/cbf.1109

[40] Mayadas, T.N., Cullere, X. and Lowell, C.A. (2014) The Multifaceted Functions of Neutrophils. Annual Review of Pathology, 9, 181-218. http://dx.doi.org/10.1146/annurev-pathol-020712-164023

[41] Wilgus, T.A., Roy, S. and McDaniel, J.C. (2013) Neutrophils and Wound Repair: Positive Actions and Negative Reactions. Advances in Wound Care, 2, 379-388. http://dx.doi.org/10.1089/wound.2012.0383

[42] Fauth, U., Schlechtriemen, T., Heinrichs, W., Puente-Gonzalez, I. and Halmagyi, M. (1993) The Measurement of Enzyme Activities in the Resting Human Polymorphonuclear Leukocyte-Critical Estimate of a Method. European Journal of Clinical Chemistry and Clinical Biochemistry, 31, 5-16.

[43] Fauth, U., Heinrichs, W., Puente-Gonzalez, I. and Halmagyi, M. (1990) Maximal Turnover Rates of Glycolysis Enzymes and of the Citrate Cycle of Separated Granulocytes in the Postoperative Period. Infusionstherapie, 17, 178-183.

[44] Jemelin, M. and Frei, J. (1970) Leukocyte Energy Metabolism. 3. Anaerobic and Aerobic ATP Production and Related Enzymes. Enzymologia Biologica et Clinica (Basel), 11, 298-323.

[45] Gabrilovich, D. (2013) The Neutrophils. New Outlook for Old Cells. 3rd Edition, Imperial College Press, London. http://dx.doi.org/10.1142/p823

[46] Break, T.J., Jun, S., Indramohan, M., Carr, K.D., Sieve, A.N., Dory, L. and Berg, R.E. (2012) Extracellular Superoxide Dismutase Inhibits Innate Immune Responses and Clearance of an Intracellular Bacterial Infection. Journal of Immunology, 188, 3342-3350. http://dx.doi.org/10.4049/jimmunol.1102341

[47] Radi, R. (2013) Peroxynitrite, a Stealthy Biological Oxidant. Journal of Biological Chemistry, 288, 26464-26472. http://dx.doi.org/10.1074/jbc.R113.472936

[48] Vitecek, J., Lojek, A., Valacchi, G. and Kubala, L. (2012) Arginine-Based Inhibitors of Nitric Oxide Synthase: Therapeutic Potential and Challenges. Mediators of Inflammation, 2012, Article ID: 318087.

[49] Wu, G., Bazer, F.W., Davis, T.A., Kim, S.W., Li, P., Marc Rhoads, J., et al. (2009) Arginine Metabolism and Nutrition in Growth, Health and Disease. Amino Acids, 37, 153-168. http://dx.doi.org/10.1007/s00726-008-0210-y

[50] Wu, G.Y. (2009) Amino Acids: Metabolism, Functions, and Nutrition. Amino Acids, 37, 1-17. http://dx.doi.org/10.1007/s00726-009-0269-0

[51] Buijs, N., Luttikhold, J., Houdijk, A.P. and van Leeuwen, P.A. (2012) The Role of a Disturbed Arginine/NO Metabolism in the Onset of Cancer Cachexia: A Working Hypothesis. Current Medicinal Chemistry, 19, 5278-5286. http://dx.doi.org/10.2174/092986712803833290

[52] Munder, M. (2009) Arginase: An Emerging Key Player in the Mammalian Immune System. British Journal of Pharmacology, 158, 638-651. http://dx.doi.org/10.1111/j.1476-5381.2009.00291.X

[53] Keough, M.P., Hayes, C.S., DeFeo, K. and Gilmour, S.K. (2011) Elevated Epidermal Ornithine Decarboxylase Activity Suppresses Contact Hypersensitivity. Journal of Investigative Dermatology, 131, 158-166. http://dx.doi.org/10.1038/jid.2010.263

[54] Lavoie-Lamoureux, A., Martin, J.G. and Lavoie, J.P. (2014) Characterization of Arginase Expression by Equine Neutrophils. Veterinary Immunology and Immunopathology, 157, 206-213. http://dx.doi.org/10.1016/j.vetimm.2013.12.007

[55] Cervelli, M., Amendola, R., Polticelli, F. and Mariottini, P. (2012) Spermine Oxidase: Ten Years after. Amino Acids, 42, 441-450. http://dx.doi.org/10.1007/s00726-011-1014-z

[56] Brooks, W.H. (2012) Autoimmune Diseases and Polyamines. Clinical Reviews in Allergy \& Immunology, 42, 58-70. http://dx.doi.org/10.1007/s12016-011-8290-y

[57] Minois, N., Carmona-Gutierrez, D. and Madeo, F. (2011) Polyamines in Aging and Disease. Aging (Albany NY), 3, 716-732.

[58] Wallace, H.M. (2009) The Polyamines: Past, Present and Future. Essays in Biochemistry, 46, 1-10. http://dx.doi.org/10.1042/bse0460001

[59] Casero, R.A. and Pegg, A.E. (2009) Polyamine Catabolism and Disease. Biochemical Journal, 421, 323-338. http://dx.doi.org/10.1042/BJ20090598

[60] Fortin, C.F., McDonald, P.P., Fulop, T. and Lesur, O. (2010) Sepsis, Leukocytes, and Nitric Oxide (NO): An Intricate Affair. Shock, 33, 344-352. http://dx.doi.org/10.1097/SHK.0b013e3181c0f068 
[61] Valenca, S.S., Rueff-Barroso, C.R., Pimenta, W.A., Melo, A.C., Nesi, R.T., et al. (2011) L-NAME and L-Arginine Differentially Ameliorate Cigarette Smoke-Induced Emphysema in Mice. Pulmonary Pharmacology \& Therapeutics, 24, 587-594. http://dx.doi.org/10.1016/j.pupt.2011.05.006

[62] Weaver, H., Shukla, N., Ellinsworth, D. and Jeremy, J.Y. (2012) Oxidative Stress and Vein Graft Failure: A Focus on NADH Oxidase, Nitric Oxide and Eicosanoids. Current Opinion in Pharmacology, 12, 160-165. http://dx.doi.org/10.1016/j.coph.2012.01.005

[63] Singh, S. and Gupta, A.K. (2011) Nitric Oxide: Role in Tumour Biology and iNOS/NO-Based Anticancer Therapies. Cancer Chemotherapy and Pharmacology, 67, 1211-1224. http://dx.doi.org/10.1007/s00280-011-1654-4

[64] Azadniv, M., Torres, A., Boscia, J., Speers, D.M., Frasier, L.M., Utell, M.J. and Frampton, M.W. (2001) Neutrophils in Lung Inflammation: Which Reactive Oxygen Species Are Being Measured? Inhalation Toxicology, 13, 485-495. http://dx.doi.org/10.1080/08958370151131855

[65] Mühling, J., Engel, J., Halabi, M., Müller, M., Fuchs, M., et al. (2006) Nitric Oxide and Polyamine Pathway-Dependent Modulation of Neutrophil Free Amino- and Alpha-Keto Acid Profiles or Host Defense Capability. Amino Acids, 31, 11-26. http://dx.doi.org/10.1007/s00726-006-0273-6

[66] Engel, J.M., Mühling, J., Weiss, S., Kärcher, B., Lohr, T., Menges, T., Little, S. and Hempelmann, G. (2006) Relationship of Taurine and Other Amino Acids in Plasma and in Neutrophils of Septic Trauma Patients. Amino Acids, 30, 87-94. http://dx.doi.org/10.1007/s00726-005-0238-1

[67] Hansen, S.H. and Grunnet, N. (2013) Taurine, Glutathione and Bioenergetics. Advances in Experimental Medicine and Biology, 776, 3-12. http://dx.doi.org/10.1007/978-1-4614-6093-0_1

[68] Buijs, N., Worner, E.A., Brinkmann, S.J., Luttikhold, J., van der Meij, B.S., Houdijk, A.P.J. and van Leeuwen, P.A.M. (2013) Novel Nutritional Substrates in Surgery. Proceedings of the Nutrition Society, 72, 277-287. http://dx.doi.org/10.1017/S0029665112003047

[69] Ripps, H. and Shen, W. (2012) Review: Taurine: A “Very Essential” Amino Acid. Molecular Vision, 18, $2673-2686$.

[70] Fink, M.P. (2007) Ethyl Pyruvate: A Novel Anti-Inflammatory Agent. Journal of Internal Medicine, 261, 349-362. http://dx.doi.org/10.1111/j.1365-2796.2007.01789.x

[71] Fink, M.P. (2007) Ethyl Pyruvate: A Novel Treatment for Sepsis. Current Drug Targets, 8, 515-518. http://dx.doi.org/10.2174/138945007780362791

[72] Fink, M.P. (2004) Ethyl Pyruvate: A Novel Treatment for Sepsis and Shock. Minerva Anestesiologica, 70, 365-371.

[73] Reade, M.C. and Fink, M.P. (2005) Bench-to-Bedside Review: Amelioration of Acute Renal Impairment Using Ethyl Pyruvate. Critical Care, 9, 556-560. http://dx.doi.org/10.1186/cc3892

[74] Mackenzie, I. and Lever, A. (2007) Management of Sepsis. British Medical Journal, 335, 929-932. http://dx.doi.org/10.1136/bmj.39346.696620.AE

[75] Kao, K.K. and Fink, M.P. (2010) The Biochemical Basis for the Anti-Inflammatory and Cytoprotective Actions of Ethyl Pyruvate and Related Compounds. Biochemical Pharmacology, 80, 151-159. http://dx.doi.org/10.1016/j.bcp.2010.03.007

[76] Das, U.N. (2006) Pyruvate Is an Endogenous Anti-Inflammatory and Anti-Oxidant Molecule. Medical Science Monitor, 12, RA79-RA84.

[77] Das, U.N. (2006) Is Pyruvate an Endogenous Anti-Inflammatory Molecule? Nutrition, 22, 965-972. http://dx.doi.org/10.1016/j.nut.2006.05.009

[78] Bates, C.M. and Lin, F. (2005) Future Strategies in the Treatment of Acute Renal Failure: Growth Factors, Stem Cells, and Other Novel Therapies. Current Opinion in Pediatrics, 17, 215-220. http://dx.doi.org/10.1097/01.mop.0000156269.48510.4e

[79] Aneja, R. and Fink, M.P. (2007) Promising Therapeutic Agents for Sepsis. Trends in Microbiology, 15, 31-37. http://dx.doi.org/10.1016/j.tim.2006.11.005

[80] Bossola, M., Pacelli, F., Rosa, F., Tortorelli, A. and Doglietto, G.B. (2011) Does Nutrition Support Stimulate Tumor Growth in Humans? Nutrition in Clinical Practice, 26, 174-180. http://dx.doi.org/10.1177/0884533611399771

[81] Nemeth, T. and Mocsai, A. (2012) The Role of Neutrophils in Autoimmune Diseases. Immunology Letters, 143, 9-19. http://dx.doi.org/10.1016/j.imlet.2012.01.013

[82] Williams, A.E. and Chambers, R.C. (2014) The Mercurial Nature of Neutrophils: Still an Enigma in ARDS? American Journal of Physiology-Lung Cellular and Molecular Physiology, 306, L217-L230. http://dx.doi.org/10.1152/ajplung.00311.2013 
Scientific Research Publishing (SCIRP) is one of the largest Open Access journal publishers. It is currently publishing more than 200 open access, online, peer-reviewed journals covering a wide range of academic disciplines. SCIRP serves the worldwide academic communities and contributes to the progress and application of science with its publication.

Other selected journals from SCIRP are listed as below. Submit your manuscript to us via either submit@scirp.org or Online Submission Portal.
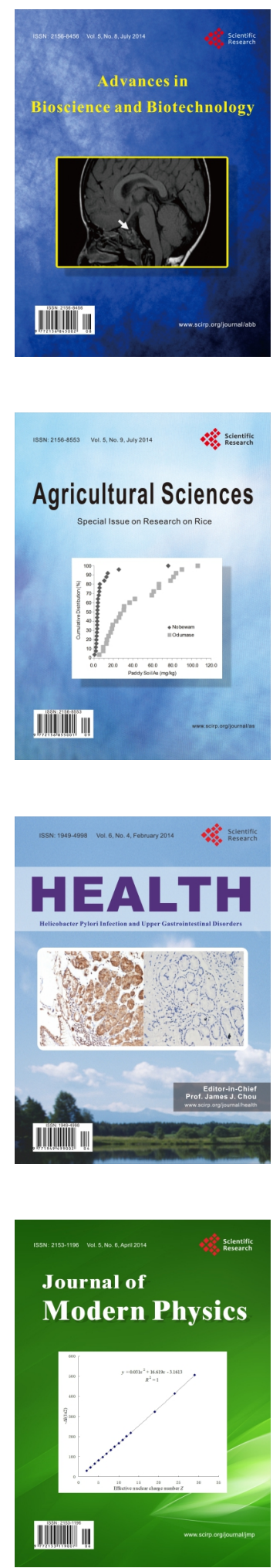
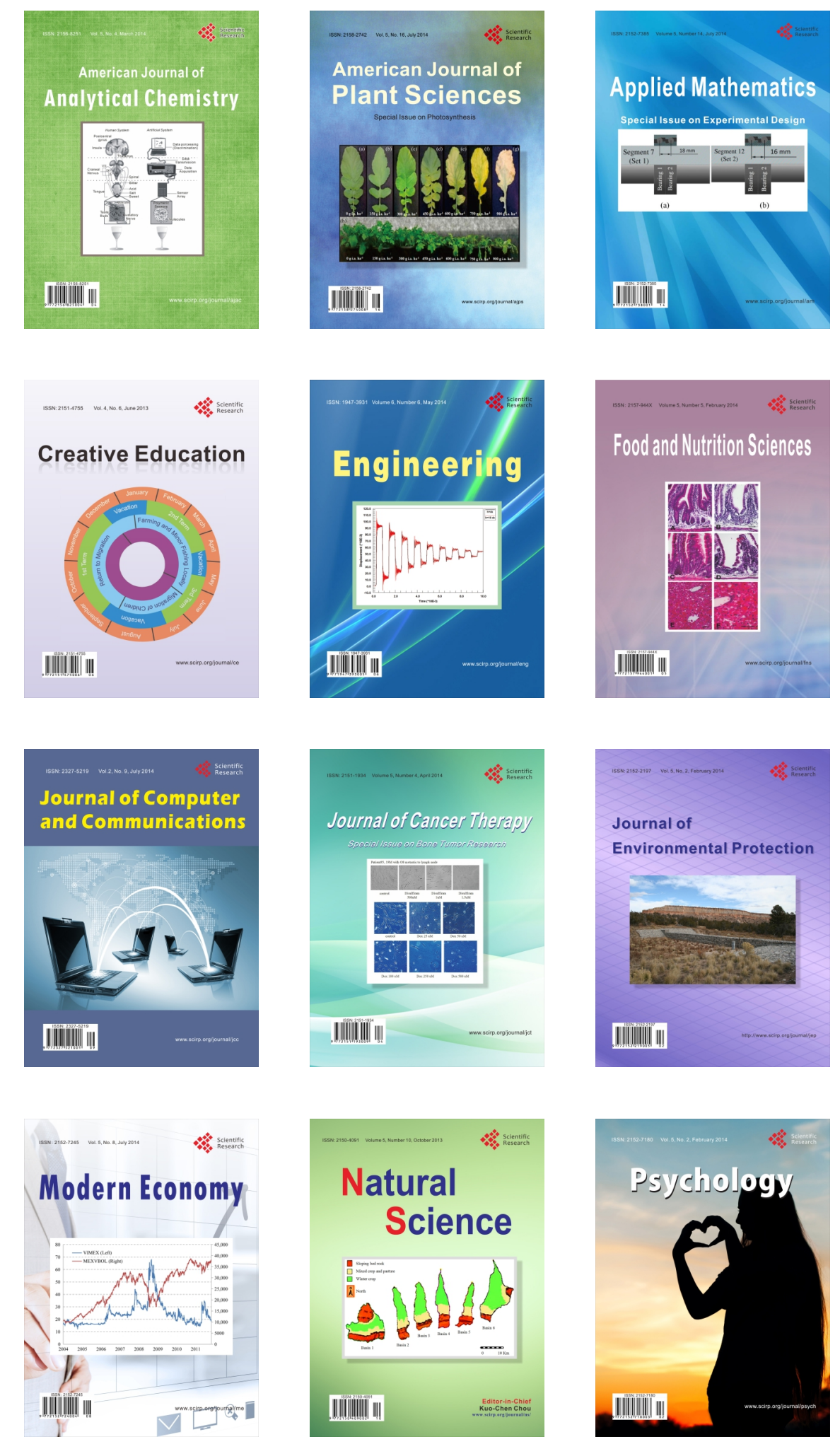